Next-generation sequencing (NGS). Technologies enabling massively parallel reading of amplified short nucleotide sequences (typically yielding hundreds of millions of reads, $100-500$ bp in length). In contrast, emerging thirdgeneration sequencing technologies read sequences without prior amplification, yielding much longer reads, albeit with reduced accuracy and throughput.

Molecular classification Classification of patient tumour samples based on molecular markers, as opposed to classification based on histomorphological appearance. *e-mail: paul.northcott @stjude.org

\title{
Medulloblastomics revisited: biological and clinical insights from thousands of patients
}

\author{
Volker Hovestadt $\mathbb{1}^{1,2}$, Olivier Ayrault $\mathbb{1}^{3,4}$, Fredrik J. Swartling $\mathbb{1}^{5}$, Giles W. Robinson $\mathbb{1}^{6}$, \\ Stefan M. Pfister (10) ${ }^{7,8,9}$ and Paul A. Northcott (10 ${ }^{10 *}$
}

Abstract | Medulloblastoma, a malignant brain tumour primarily diagnosed during childhood, has recently been the focus of intensive molecular profiling efforts, profoundly advancing our understanding of biologically and clinically heterogeneous disease subgroups. Genomic, epigenomic, transcriptomic and proteomic landscapes have now been mapped for an unprecedented number of bulk samples from patients with medulloblastoma and, more recently, for single medulloblastoma cells. These efforts have provided pivotal new insights into the diverse molecular mechanisms presumed to drive tumour initiation, maintenance and recurrence across individual subgroups and subtypes. Translational opportunities stemming from this knowledge are continuing to evolve, providing a framework for improved diagnostic and therapeutic interventions. In this Review, we summarize recent advances derived from this continued molecular characterization of medulloblastoma and contextualize this progress towards the deployment of more effective, molecularly informed treatments for affected patients.

Medulloblastoma (MB), a cerebellar tumour, is one of the most common malignant central nervous system (CNS) tumours in children (total of $\sim 440$ new diagnoses/year in the USA, $>65 \%$ diagnosed in children younger than 16 years old ${ }^{1}$ ) and a leading cause of cancer-related death in this age group ${ }^{2}$. The incidence of MB does not appear to be significantly influenced by geographical location or ancestry $y^{3,4}$. During the past two decades, large-scale genomic efforts have helped disentangle the molecular basis of $\mathrm{MB}$, including biologically and clinically relevant intertumoural heterogeneity. Four consensus molecular subgroups - WNT, Sonic hedgehog (SHH), Group 3 and Group 4, each characterized by distinct -omic (that is, genomic, epigenomic, transcriptomic and proteomic) and clinical features - are now widely recognized ${ }^{5}$. As a direct consequence, mechanistic, developmental and preclinical studies are currently being undertaken in a manner that is cognizant of molecular subgroup status. Moreover, clinical protocols have adopted molecular subgrouping strategies into routine diagnosis, treatment stratification and patient selection for molecularly targeted therapies.

In 2012, following the formal recognition of consensus molecular subgroups ${ }^{5}$, the initial wave of nextgeneration sequencing (NGS) studies conducted on primary MB samples were reported ${ }^{6-10}$. Since then, additional molecular characterization has ensued on increasingly larger cohorts, resulting in the generation of a wealth of multidimensional -omics data. As a result, the molecular classification of MB has evolved beyond the four consensus subgroups to include additional subdivisions (also referred to as 'subtypes'), and new methods for robust and accurate assignment of patients into the relevant subtypes have become mainstream. More recently, spatial and single-cell genomic approaches have been applied, delving into the intratumoural heterogeneity, cellular composition and developmental origins of $\mathrm{MB}$ at single-cell resolution. We recently reviewed the epidemiological, biological and therapeutic characteristics of $\mathrm{MB}$ in a report that was intended to be all-encompassing of the human disease ${ }^{11}$. In this Review, we emphasize what has been learned from the -omic analyses of patients with $\mathrm{MB}$ since the first NGS studies ${ }^{6}$, highlighting insights into the molecular and biological mechanisms underlying $\mathrm{MB}$ heterogeneity and the translational implications emerging from these efforts.

\section{Molecular classification}

WHO consensus subgroups and subtypes within subgroups. The first gene expression array study confirmed that MB was molecularly distinct from other embryonal brain tumours, such as primitive neuroectodermal tumour (PNET) and atypical teratoid rhabdoid tumour ${ }^{12}$. In this seminal report, published in 2002, a gene expression comparison of MB types with classic histology versus desmoplastic/nodular histology identified specific 
Intratumoural heterogeneity The observation that tumours comprise distinct malignant and non-malignant (cells of the microenvironment) cell types. The heterogeneity of malignant cells encompasses genetic heterogeneity (for example different genetic subclones) and transcriptional

heterogeneity (for example, malignant cell states resembling normal development)

Primitive neuroectodermal tumour defined, poorly differentiated childhood brain tumours. More recently, PNET has been of both novel and previously known brain tumour entities by molecular profiling.

Atypical teratoid rhabdoid tumour

A rare and highly malignant type of childhood brain tumour that is characterized by mutations in the SMARCB 1 gene.

Classic histology

The most common

histological variant found in

all medulloblastoma subgroups,

characterized by densely

packed small round or

oval cells and a high nuclear:

cytoplasmic ratio.

Desmoplastic/nodular histology

A histological variant mostly restricted to Sonic hedgehog medulloblastoma, characterized by a varying number of islands of neurocytic differentiation and internodular desmoplasia.
(PNET). A class of histologically reclassified into a number

upregulation of genes involved in Hedgehog signalling, including patched homologue 1 (PTCH1), GLI1 and $M Y C N$, in desmoplastic tumours ${ }^{12}$. A parallel report comparing the expression profiles of a series of genetically engineered mouse (GEM) models with normal cerebellar controls identified similar activation of Hedgehog pathway-associated gene sets in MB GEM models ${ }^{13}$. Together, these early reports of the gene expression signatures defining MBs in humans and mice paved the way for the molecular era that followed. Multiple independent gene expression array profiling studies conducted on patient cohorts subsequently described distinct molecular subgroups within MB that differed in their demographics, genetic alterations and clinical outcomes ${ }^{14-17}$, culminating in the definition of consensus subgroups ${ }^{5}$. The WNT and SHH subgroups, which represent approximately $10 \%$ and $30 \%$, respectively, of all patients with $\mathrm{MB}$, are associated with activation of the WNT and $\mathrm{SHH}$ signalling pathways. The WNT and $\mathrm{SHH}$ subgroups are discrete across studies and technologies, providing a basis for their incorporation into the 2016 update of the WHO Classification of Central Nervous System Tumours ${ }^{18}$. Further subdivision within the $\mathrm{SHH}$ subgroup according to TP53 mutation status (SHH with wild-type TP53 and SHH with mutant TP53) is also recognized by the WHO. The Group 3 and Group 4 subgroups, which represent the remaining $25 \%$ and $35 \%$, respectively, of all patients with $\mathrm{MB}$, exhibit some molecular and biological similarities (detailed below). Consequently, these subgroups are formally recognized by the WHO as non-WNT/nonSHH MB, and the Group 3 and Group 4 designations are listed as provisional entities.

Differential expression analysis between MB subgroups has led to the identification of select biomarkers that enable molecular classification based on immunohistochemistry ${ }^{16,19,20}$, the NanoString gene expression assay $^{21,22}$ and other panel-based gene expression assays ${ }^{23}$. While these assays are widely accessible in clinical practice, DNA methylation arrays, which can measure hundreds of thousands of methylation sites across the genome, have emerged as the platform of choice for $\mathrm{MB}$ classification. Owing to the relative stability of DNA, methylation arrays and derivative-targeted DNA methylation-based assays allow for the routine analysis of clinical samples in a diagnostic setting as well as

\footnotetext{
Author addresses

${ }^{1}$ Department of Pathology and Center for Cancer Research, Massachusetts General Hospital and Harvard Medical School, Boston, MA, USA.

${ }^{2}$ Broad Institute of Harvard and MIT, Cambridge, MA, USA.

${ }^{3}$ Institut Curie, PSL Research University, CNRS UMR, INSERM, Orsay, France. ${ }^{4}$ Université Paris Sud, Université Paris-Saclay, CNRS UMR 3347, INSERM U1021,

Orsay, France.

${ }^{5}$ Department of Immunology, Genetics and Pathology, Science for Life Laboratory, Rudbeck Laboratory, Uppsala University, Uppsala, Sweden.

${ }^{6}$ Department of Oncology, St Jude Children's Research Hospital, Memphis, TN, USA.

${ }^{7}$ Hopp Children's Cancer Center Heidelberg (KiTZ), Heidelberg, Germany.

${ }^{8}$ Division of Paediatric Neurooncology, German Cancer Research Center (DKFZ) and German Cancer Consortium (DKTK), Heidelberg, Germany.

${ }^{9}$ Department of Paediatric Haematology and Oncology, Heidelberg University Hospital, Heidelberg, Germany.

${ }^{10}$ Department of Developmental Neurobiology, St Jude Children's Research Hospital, Memphis, TN, USA
}

the profiling of archival tumour material, with limited technical variation between institutions ${ }^{24-28}$.

In 2017, three independent studies ${ }^{29-31}$ used DNA methylation analysis or combined DNA methylation and gene expression analysis to investigate the additional substructure within subgroups, leading to varying definitions of molecular subtypes across studies (FIG. 1; discussed further below).

WNT medulloblastoma. WNT MB occurs primarily in children from 4 years of age to early adulthood (median age of diagnosis $\sim 11$ years) and exhibits a balanced male:female ratio (FIG. 2). WNT-MB tumours are usually of classic histology and are infrequently metastatic at diagnosis. The outcomes for patients with WNT $\mathrm{MB}$ are favourable, with 5-year survival of $95 \%$ or bet$\operatorname{ter}^{20,32-35}$. Somatic mutations in CTNNB1 (which encodes $\beta$-catenin) are the hallmark genetic event defining this subgroup ( $\sim 85 \%$ of patients) ${ }^{17,34}$, with most of the remaining patients typified by pathogenic germline variants in adenomatous polyposis coli $(A P C)^{36}$. WNT-MB genomes are mostly devoid of somatic copy-number alterations (SCNAs), except for loss of one copy of chromosome 6 (that is, monosomy 6) in most patients ${ }^{9,30}$.

WNT MB has been described as largely homogeneous between patients, with regard to genome-wide expression and methylation profiles. However, two molecular subtypes, WNT- $\alpha$ and WNT- $\beta$, have been suggested that differ in age at diagnosis (median age of 10 versus 20 years, respectively) and frequency of monosomy 6 (REF. ${ }^{29}$ ). The survival outcomes of adults with WNT MB (that is, WNT- $\beta^{29}$ ) have been inconsistent in the literature, with some reports describing outcomes as comparably favourable, relative to those of paediatric patients with WNT $\mathrm{MB}^{29,37}$, and others reporting reduced overall survival ${ }^{35}$

SHH medulloblastoma. SHH MB displays an intriguing bimodal age distribution, representing the most common molecular subgroup in both infants $(<3$ years of age) and adults ( $>17$ years of age), with fewer cases being diagnosed during childhood and adolescence (FIG. 2). Demographically, SHH MB is more common in males than in females (approximately 2:1). Classic and desmoplastic/nodular (including MB with extensive nodularity) histologies occur at similar frequencies (each accounting for $\sim 40 \%$ of patients), with a largecell/anaplastic (LCA) histology making up the remain$\operatorname{der}^{20,32}$. Mutations and focal SCNAs targeting genes in the $\mathrm{SHH}$ signalling pathway represent the most common genetic events, including inactivating germline or somatic mutations and deletions of PTCH1 and of suppressor of fused homologue (SUFU), activating mutations in smoothened homologue $(S M O)$ and amplifications of GLI2 ( $\mathrm{REFS}^{30,38}$ ). Frequent chromosomal alterations include loss of chromosomes $9 \mathrm{q}, 10 \mathrm{q}, 14 \mathrm{q}$ and $17 \mathrm{p}$, as well as gain of chromosomes 2 and $9 p^{9,30}$.

Age-associated molecular differences discriminating infant and adult SHH MB have been observed through both gene expression and DNA methylation array profiling $^{31,38,39}$. Compared with their paediatric counterparts, adult SHH MBs are characterized by a higher overall 

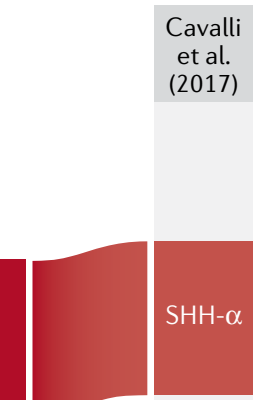

SHH- $\beta$

紊
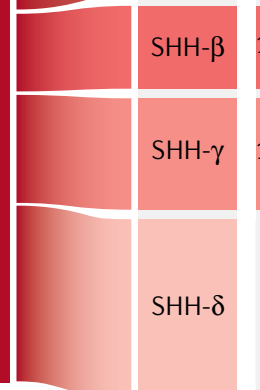

\section{Kool}

et al.

(2014)

$\stackrel{\stackrel{ \pm}{\frac{0}{0}} \frac{ \pm}{5}}{\frac{5}{5}}$
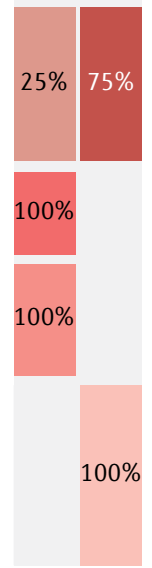
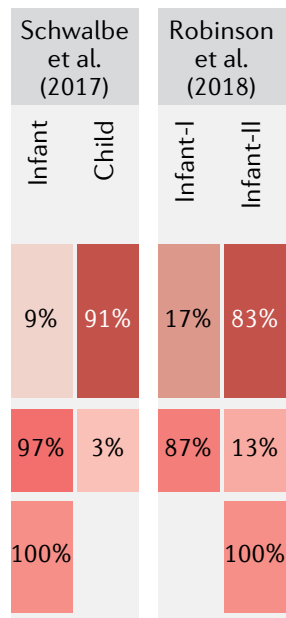

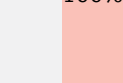

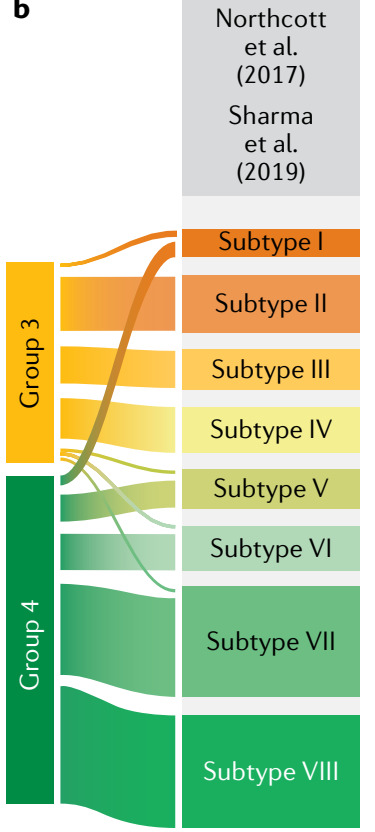

Schwalbe et al.

(2017)

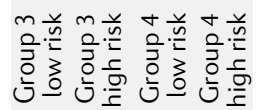

$\begin{array}{lll}50 \% & 17 \% & 33 \%\end{array}$

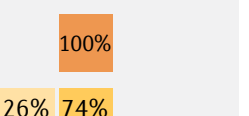

$26 \% 74 \%$

$100 \%$

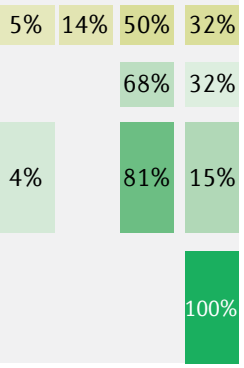

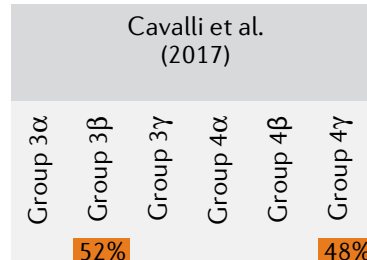

$48 \%$

$32 \% \quad 68 \%$

\begin{tabular}{l|ll}
$84 \%$ & $13 \%$ & $3 \%$
\end{tabular}

$97 \% \quad 3 \%$

$3 \%$

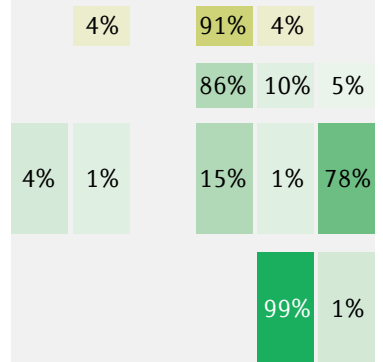

Fig. 1 | Comparison of MB DNA methylation-derived subtypes described across recent studies. a $\mid$ Correspondence between the four molecular subtypes of the Sonic hedgehog medulloblastoma (SHH-MB) subgroup, described by Cavalli et al. ${ }^{29}$ ( $n=223$ samples) and the subtypes described in three additional studies (Kool et al. ${ }^{38}, n=129$ samples; Schwalbe et al. ${ }^{31}, n=103$ samples; Robinson et al. ${ }^{44}, n=82$ samples). DNA methylation profiles from all samples of each additional study were used to classify patients into the four molecular subtypes using a machine-learning approach. The height of each row corresponds to the fraction of samples per subtype in the Cavalli et al. study ${ }^{29}(\mathrm{SHH}-\alpha=29 \% ; \mathrm{SHH}-\beta=16 \% ; \mathrm{SHH}-\gamma=21 \%$; SHH- $\delta=34 \%)$. Percentages indicate overlap of the predicted subtypes with the original subtype annotations in each additional study. The samples from the study by Robinson et al. ${ }^{44}$ were not predicted as $\mathrm{SHH}-\delta$, consistent with the study including only patients under the age of 6 years. $\mathbf{b} \mid$ A similar comparison of the eight molecular subtypes of Group 3 and Group 4 MB described by Northcott et al..$^{30}$ and Sharma et al..$^{134}(n=1,370$ samples; subtype: $I=4 \%, I I=13 \%, I I I=9 \%, I V=10 \%, V=8 \%, V I=9 \%, V I I=22 \%$, $\mathrm{VIII}=25 \%$ ) and the subtypes described in two additional studies (Schwalbe et al. ${ }^{31}, n=273$ samples; Cavalli et al. ${ }^{29}, n=470$ samples). The line widths between the two consensus subgroups (Group 3 and Group 4) and the eight DNA methylation subtypes indicate the fraction of samples per subtype that were originally classified as Group 3 or Group 4 MB.

mutational burden, a higher prevalence of $\mathrm{SHH}$ pathwayassociated mutations (including higher incidence of PTCH1 and SMO alterations) and a more expansive list of chromatin modifier mutations (that is, in bromodomain and PHD finger containing 1 (BRPF1) and CREBbinding protein $(C R E B B P)$ ); virtually all such MBs also harbour telomerase reverse transcriptase (TERT) promoter mutations $s^{38,40-42}$. Intriguingly, transcriptional comparison of tumours between available SHH-MB mouse models and patients has suggested that, despite the mouse tumours being primarily driven by inactivation of Ptch 1 or activation of $S m o$ - genetic events that occur in both paediatric and adult tumours - the current models are more molecularly similar to adult $\mathrm{SHH} \mathrm{MB}{ }^{43}$.

More recently, four molecular subtypes of SHH MB have been reported ${ }^{29}$ (FICS 1 1, 2): $\mathrm{SHH}-\beta$ and $\mathrm{SHH}-\gamma$ correspond to infant subtypes (median ages of diagnosis 1.9 and 1.3 years, respectively), whereas $\mathrm{SHH}-\alpha$ and SHH- $\delta$ correspond to childhood/adolescent and adult subtypes, respectively (median ages of diagnosis 8 and 26 years, respectively). Subtype $\mathrm{SHH}-\alpha$ is enriched for patients harbouring TP53 mutations ( $\sim 1 / 3$ of patients with $\mathrm{SHH}-\alpha)$ and is associated with inferior outcomes, compared with patients with $\mathrm{SHH}-\delta$. Infant subtype $\mathrm{SHH}-\beta$ showed a lower 5 -year survival than $\mathrm{SHH}-\gamma$. Similar observations were made in an independent study reporting on a clinical trial in infants and young children (under 6 years of age) that identified two molecular subtypes, iSHH-I (equivalent to SHH- $\beta$ ) and iSHH-II (equivalent to $\mathrm{SHH}-\gamma$ and $\mathrm{SHH}-\alpha)^{44}$.

Group 3 and Group 4 medulloblastoma. Group $3 \mathrm{MB}$ occurs during infancy and childhood and is rarely seen in patients older than 18 years of age, whereas Group $4 \mathrm{MB}$ occurs across all age groups (FIG. 2). The male:female ratios are 2:1 or higher for both subgroups. LCA histology is more prevalent in Group 3 than in Group 4, and $30-40 \%$ of patients are metastatic at diagnosis in both subgroups ${ }^{32}$. MYC amplification is a common genetic feature of Group $3 \mathrm{MB}$ and is associated with a particularly poor clinical outcome ${ }^{14,16}$. MYCN and cyclin-dependent kinase 6 (CDK6) amplifications are notable genetic alterations seen in Group $4 \mathrm{MB}^{9,30}$. Isochromosome $17 \mathrm{q}$ (that is, $\mathrm{i} 17 \mathrm{q}$ ) is a hallmark cytogenetic event in both subgroups, found in $>50 \%$ of patients from either subgroup ${ }^{9,30}$.

The definitions and substructures of Group 3 and Group $4 \mathrm{MBs}$ have been a topic of debate since their initial discovery ${ }^{14-17}$. Indeed, early nomenclature did not always define Group 3 and Group 4 into distinct subgroups and in some cases described them as a single 'mixed' subgroup of patients designated as 
Craniospinal axis irradiation (CSI). Standard therapy for patients with medulloblastoma following surgery, to reduce risk of tumour regrowth and metastatic dissemination. The application of CSI is also associated with neurological impairments and secondary malignancies.

Non-synonymous mutations Genetic alterations that alter the amino acid sequence of an affected protein, possibly altering protein function. Most described recurrent mutations in medulloblastoma are non-synonymous mutations.

BTB-Kelch protein family Family of proteins

characterized by the presence of an N-terminal BTB domain and C-terminal Kelch motifs. The BTB domain facilitates protein binding, and the Kelch motifs associate to form a $\beta$-propeller facilitating proteinprotein interactions. This family function as adaptor proteins that link cullin-RING ligases to substrates for ubiquitylation.

Pineal parenchymal tumours of intermediate differentiation

(PPTID). A very rare tumour type of intermediate grade arising from the pineal parenchyma.
'non-WNT/non-SHH' MB ${ }^{20}$. However, the recognition of Group 3 and Group $4 \mathrm{MB}$ has continued to evolve in recent years, with the majority of the neuro-oncology community, and the $\mathrm{WHO}^{18}$, acknowledging their definition as mostly discrete molecular entities and supporting the utility of their distinction, as is supported in the literature (see $\mathrm{e}^{11,45-47}$ for recent reviews).

More recently, three independent studies have identified a varying number of subtypes within Group 3 and Group 4 (FIG. 1 b). Schwalbe and colleagues identified four molecular subtypes in Group 3 and Group $4 \mathrm{MB}$ that split each subgroup into high- and low-risk subtypes $^{31}$. Cavalli and colleagues identified three molecular subtypes in each subgroup: Group $3 \alpha, \beta$ and $\gamma$, and Group $4 \alpha, \beta$ and $\gamma^{29}$. In a combined analysis of Group 3 and Group 4 cases, we identified eight molecular subtypes, designated I to VIII ${ }^{30}$. To harmonize these subtype definitions, a joint analysis of 1,501 Group 3 and Group 4 MBs from all three studies was recently undertaken ${ }^{48}$. In this analysis, we showed that separation into largely the same eight subtypes best unified the substructures observed in the aforementioned studies (FIG. 1 b).

Subtype I represents the least common subtype and comprises a mix of Group 3 and Group 4 tumours (FIG. 2). Subtype I tumour genomes are generally balanced and enriched for amplification of the orthodenticle homeobox 2 (OTX2) oncogene and activation of growth factor independent 1 (GFI1) or GFI1B, which encode transcriptional repressors (described in detail below). Subtypes II, III and IV are bona fide Group 3 subtypes. Subtypes II and III are characterized by amplification of the MYC oncogene and are associated with poor outcomes. In comparison to other subtypes, Subtype IV is enriched for younger patients (median age of diagnosis 3 years) and is associated with a favourable outcome in non-infants; the low progression-free survival observed in infant patients with Subtype IV suggests that survival is dependent on treatment with craniospinal axis irradiation (CSI $)^{44}$. Subtypes V, VI and VII consist mostly of Group $4 \mathrm{MBs}$ but also include some Group 3 tumours. Subtype V genomes are characterized by amplification of both $M Y C$ and $M Y C N$ and are associated with modest outcomes. Subtype VIII is the most common and only pure Group 4 subtype, mostly occurring in older children (median age of diagnosis 10 years). Subtype VIII tumours display a balanced genome, except for the presence of $\mathrm{il7q}$ in most cases. Subtype VIII is associated with favourable 5-year survival; however, many patients are affected by late relapse and death, a feature that is unique to this subtype ${ }^{48}$.

\section{Medulloblastoma genomic analyses}

Gene mutations. MB NGS studies have primarily detailed somatic non-synonymous mutations affecting protein-coding genes in relatively modest patient cohorts $^{7,8,10,49}$. New recurrently mutated genes emerged from these analyses, including DEAD-box helicase $3 \mathrm{X}$ linked $(D D X 3 X)$, BCL-6 co-repressor (BCOR), CTD nuclear envelope phosphatase 1 (CTDNEP1), and T-box brain transcription factor 1 (TBR1), among others. In addition, genes encoding chromatin modifiers such as the lysine methyltransferases KMT2D (also known as $M L L 2$ ) and $K M T 2 C$ (also known as $M L L 3$ ), the lysine demethylase KDM6A, and SWI/SNF-related matrixassociated actin-dependent regulator of chromatin subfamily A member 4 (SMARCA4), previously identified by large-scale exome resequencing ${ }^{50}$, were also confirmed, and their mutational frequencies and distributions were contextualized by subgroup. Despite these advances, the initial standalone studies were underpowered to adequately detail the broader scope of the low-frequency driver gene alterations that contribute to $\mathrm{MB}$, especially in Groups 3 and 4, which were heterogeneous and devoid of highly recurrent protein-coding gene mutations ${ }^{6}$.

Recently, an international collaborative effort, co-led by authors of this Review, towards comprehensive characterization of the MB genomic landscape summarized putative driver gene alterations across a series of 491 primary MB samples ${ }^{30}$. As expected, the WNT and $\mathrm{SHH}$ $\mathrm{MB}$ subgroups were largely characterized by mutations and SCNAs affecting known genes. Of interest, functional annotation of recurrently altered genes identified somatic deregulation of SWI/SNF family chromatinremodelling genes (namely, SMARCA4, ARID1A and ARID2) in one-third of WNT-MB cases, and recurrent targeting of histone acetyltransferase genes (namely, CREBBP, KANSL1, BRPF1 and others) in nearly $20 \%$ of SHH MBs. Detailed mechanistic studies will be required in order to determine how somatic targeting of these chromatin-associated complexes potentiates MB pathogenesis in the affected subgroups.

In Group 3 and Group $4 \mathrm{MBs}$, the multidimensional molecular analysis performed on this broader set of patients ( $n=324$ total) confirmed that recurrent protein-coding gene mutations remained relatively rare. SMARCA 4 mutations occurred in $9 \%$ of Group 3 and only $2 \%$ of patients from Group 4. KDM6A (7\%), zinc finger MYM-type containing 3 (ZMYM3) (6\%) and KMT2C (6\%) represented the most commonly mutated genes in Group 4. Previously unknown somatic in-frame insertions that affected Kelch repeat and BTB domain containing 4 (KBTBD4) were evenly distributed between Group 3 and Group 4 MBs (6\% of patients from either subgroup). Although it is poorly characterized to date, KBTBD4 is a member of the BTB-Kelch protein family and is predicted to recruit protein substrates to cullinRING ligases for targeted ubiquitylation and protein degradation $^{51}$. MB-associated KBTBD4 insertions were confined to the Kelch domain and were deemed unlikely to disrupt the overall domain structure but instead converged on the known substrate-binding interface described for other BTB-Kelch protein family members. Recently, somatic in-frame insertions identical to those seen in MB have been reported in pineal parenchymal tumours of intermediate differentiation $(\mathrm{PPTID})^{52}$, suggesting that common oncogenic mechanisms may be shared between patients affected by $\mathrm{MB}$ and PPTID.

Structural alterations and enhancer hijacking. The Group 3 and Group $4 \mathrm{MB}$ genomes are characterized by a preponderance of SCNAs and structural variants $(\mathrm{SVs})^{9}$, suggesting that these alterations play an integral role 


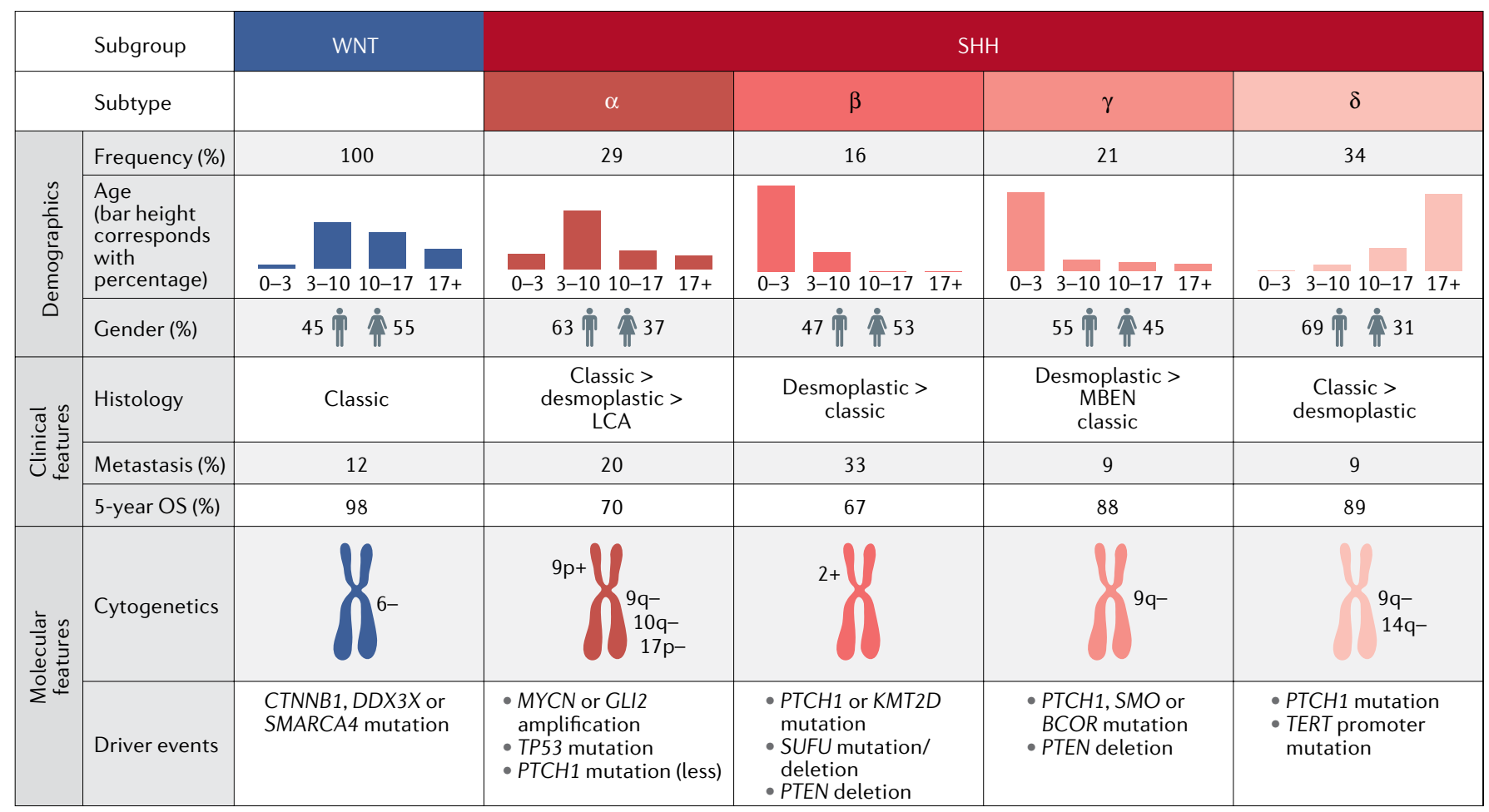

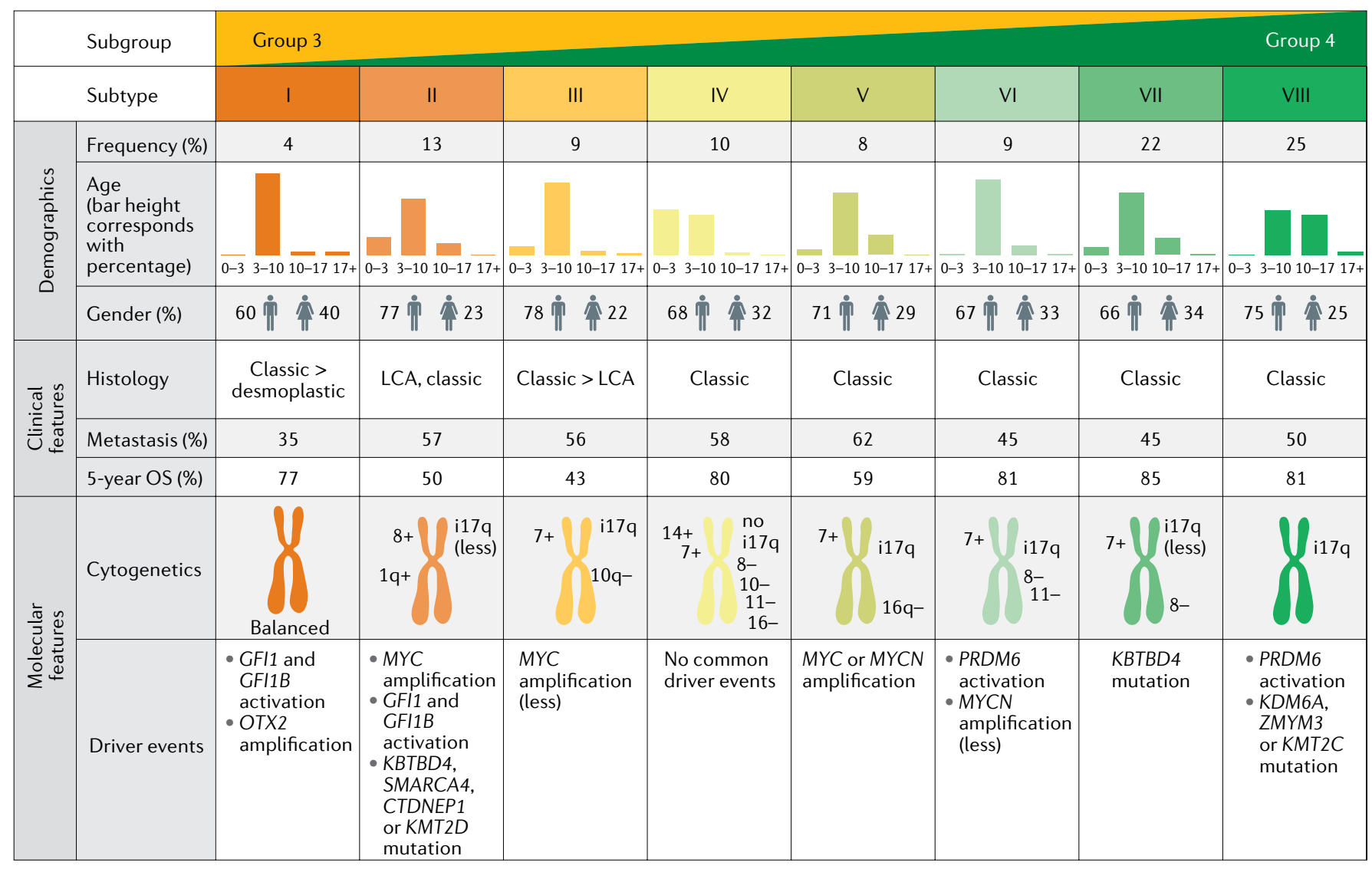

in disease pathogenesis. Analysis of the genomes of 137 Group 3 and Group 4 MB samples identified a series of atypical SVs (that is, deletions, duplications, inversions and more complex genomic alterations) mapping to chromosome $9 q 34$ that were specific to these subgroups ${ }^{53}$. Integration with sample-matched gene expression data uncovered pronounced, SV-associated upregulation of GFI1B in affected samples. GFI1B is a transcriptional repressor that is primarily known for its role in $\mathrm{T}$ cell and B cell development, as well as in 
Fig. 2 | Summary of demographic, clinical and molecular features of novel MB subtypes. Values for age and gender distribution, frequency of metastasis, and 5-year overall survival (OS) for the WNT and Sonic hedgehog (SHH) subgroups are derived from the Cavalli et al. study ${ }^{29}$. Driver events were additionally derived from the Kool et al. ${ }^{38}$ and Robinson et al. ${ }^{44}$ studies. Similarly, values for the Group 3 and Group 4 subgroups of medulloblastoma (MB) were derived from the Sharma et al. study ${ }^{134}$. BCOR, BCL-6 co-repressor; CTDNEP1, CTD nuclear envelope phosphatase 1; CTNNB1, $\beta$-catenin; DDX3X, DEAD-box helicase 3X-linked; GFI1, growth factor independent 1 transcriptional repressor; i17q, isochromosome 17q; KBTBD4, Kelch repeat and BTB domain containing 4; KDM6A, lysine demethylase $6 \mathrm{~A} ; \mathrm{KMT2C}$, lysine methyltransferase $2 \mathrm{C}$; LCA, large-cell/ anaplastic; MBEN, MB with extensive nodularity; OTX2, orthodenticle homeobox 2; PRDM6, PR/SET domain 6; PTCH1, patched homologue 1; SMARCA4, SWI/SNF-related matrix-associated actin-dependent regulator of chromatin subfamily A member 4; SMO, smoothened homologue; SUFU, suppressor of fused homologue; TERT, telomerase reverse transcriptase; ZMYM3, zinc finger MYM-type containing 3.

haematopoietic malignancies in which it functions as an oncogene $e^{54}$. The related family member GFI1 was also determined to be aberrantly expressed in a mutually exclusive set of Group 3 and Group 4 MBs harbouring SV breakpoints proximal to the GFI1 locus. Using an orthotopic transplantation approach, GFI1B and GFI1 were validated as novel MB oncogenes capable of cooperating with MYC to promote highly aggressive Group 3-like $\mathrm{MB}$ in mice ${ }^{53}$. Histone chromatin immunoprecipitation followed by sequencing (ChIP-seq) data for histone $\mathrm{H} 3$ lysine 27 acetylation $(\mathrm{H} 3 \mathrm{~K} 27 \mathrm{ac})$ that marked active enhancers suggested that the activation of GFI1B and GFI1 expression in MB was accomplished via SV-dependent misappropriation of distal, highly active enhancers or super-enhancers to their normally repressed gene promoters. Similar to classical translocations that lead to overexpression of established oncogenes, such as $\operatorname{Ig} G-M Y C$ in Burkitt lymphoma ${ }^{55,56}$, this mechanism of SV-dependent gene activation was designated 'enhancer hijacking' and has since been explored and documented in numerous follow-up studies in other cancer types ${ }^{57-61}$. Overall, enhancer hijacking-associated GFI1 and GFI1B activation is estimated to account for $\sim 12-15 \%$ of patients with Group 3 and Group $4 \mathrm{MB}$, with clear enrichment of these events in Subtype I, and to a lesser extent in Subtype $\mathrm{II}^{30}$.

Motivated by the discovery of enhancer hijacking, a novel computational pipeline, termed cis-expression structural alteration mapping $(\mathrm{CESAM})^{60}$, was developed to systematically identify additional enhancer-hijacking events through the integration of SV breakpoints and gene expression data. By applying CESAM to samplematched genomic datasets derived from 164 patients with MB, we discovered PRDM6 as a novel target of enhancer hijacking in $17 \%$ of patients with a Group 4 diagnosis $^{30}$. PRDM6 maps to chromosome 5q23, approximately $600 \mathrm{~kb}$ downstream of SNCAIP - a locus known to be targeted by highly recurrent, stereotypical tandem duplications exclusively in Group $4 \mathrm{MB}^{9}$. Through multi-omic data integration for a series of Group $4 \mathrm{MBs}$, a putative model of enhancer hijacking-mediated activation of PRDM6 was proposed. PRDM6 is described as a transcriptional repressor, mediating gene silencing through intrinsic histone $\mathrm{H} 4$ lysine 20 (H4K20) methyltransferase activity, in concert with known chromatin-associated, repressive complexes ${ }^{62,63}$. To date, PRDM6 represents the most frequent somatically altered gene in Group 4
$\mathrm{MB}$, which, together with mutations targeting other chromatin-modifying genes, further implicates deregulation of physiological transcriptional control as an essential mechanism underlying Group $4 \mathrm{MB}$ pathogenesis.

\section{Transcriptomic and epigenomic analyses}

Compared with childhood leukaemias ${ }^{64}$ and other paediatric brain cancers (for example, supratentorial ependymoma $^{65}$ and pilocytic astrocytoma ${ }^{66}$ ), recurrent gene fusions are rare in $\mathrm{MB}$. Early transcriptome sequencing discovered recurrent $P V T 1$ gene fusions in Group $3 \mathrm{MB}$ that were linked to chromothripsis and MYC amplification on chromosome 8q24 (REF.'). Additional PVT1-associated fusion events have since been reported in Group 3, including PVT1-NDRG1, PVT1-LINC00964, PVT1$Z C H 3$ and others ${ }^{30}$. PVT1 encodes a long intergenic non-coding RNA (lincRNA) harbouring a cluster of six annotated microRNAs (miRNAs; namely, miR-1204, miR-1205, miR-1206, miR-1207-5p, miR-1207-3p, and miR-1208). Several reports have linked PVT1 overexpression and activity with pro-tumorigenic phenotypes $^{67-73}$. In contrast, a recent study suggested that the PVT1 gene promoter is a tumour suppressor DNA element that inhibits MYC expression through enhancerpromoter competition in $\mathrm{cis}^{74}$. Future mechanistic and phenotypic studies in relevant model systems will be necessary to decipher the role of the PVT1 fusions, as well as other fusions, seen in MB.

Paired DNA methylation and transcriptome analyses of patient samples with $\mathrm{MB}$ have also led to the identification of alternate promoters, indicating that transcriptional initiation of specific genes might reside outside previously annotated promoter regions. Analysis of highcoverage RNA sequencing (RNA-seq) data in $43 \mathrm{MB}$ samples revealed 262 novel first exons that were spliced to internal exons in excess of $15 \mathrm{~kb}$ upstream of the previously annotated transcriptional start sites (TSSs), with some being located more than $500 \mathrm{~kb}$ away ${ }^{75}$. Many of these alternate transcripts were expressed in a subgroupspecific manner, which often coincided with patterns of differential DNA methylation in the region of the novel TSS. One notable example is the pluripotency factor $L I N 28 B$, which has been described as regulating multiple oncogenic processes, including downregulation of the tumour-suppressive let-7 miRNA family ${ }^{76}$. In MB, LIN28B is expressed specifically in Group 3 and Group 4 MBs. In about half of Group 3 cases and nearly all Group 4 cases, the annotated promoter region is fully hypermethylated, and transcription is initiated at a novel first exon that is spliced to the second annotated exon.

Systematic miRNA profiling of patient samples resulted in the identification of a number of differentially expressed miRNAs, both between MB subgroups and relative to normal controls ${ }^{14,77,78}$. Among the best studied is the oncogenic miR-17-92 cluster, which was described as being genetically amplified and overexpressed specifically in $\mathrm{SHH} \mathrm{MB} \mathrm{MB}^{77,79}$ and as being required for the formation of $\mathrm{SHH} \mathrm{MB}$ in the Ptch $1^{+/-}$ mouse MB model ${ }^{80}$. Furthermore, locked nucleic acidmediated silencing of miR-17-92 prolonged the survival of mice with intracranial SHH-MB allografts ${ }^{81}$. Another well-studied example is the miR-183-96-182 cluster, 
which is highly expressed in WNT, Group 3 and Group 4 $\mathrm{MB}$, targeting the AKT-PI3K-mTOR pathway and regulating cell proliferation and migration both in vitro and in vivo ${ }^{82-84}$. Other miRNAs are downregulated in MB and have been described as tumour suppressors, including miR-125b, miR-326 and miR-324-5p, which have been described to regulate the SHH signalling pathway ${ }^{85}$, and miR-124a and miR-9, which regulate both the RE1-silencing transcription factor (REST) complex and cell proliferation ${ }^{86-89}$. An extensive review of the role of miRNAs in MB has recently been published ${ }^{90}$.

Genome-wide analysis of differential DNA methylation between MB subgroups and control tissues showed that the classical notion of gene silencing through promoter hypermethylation was not a prominent feature in $\mathrm{MB}^{75}$. The most abundant pattern of differential methylation was identified in regions extending several kilobases downstream of the promoter into the gene body, in which hypomethylation correlated with elevated gene expression (in promoter downstream correlated regions, pdCRs). About $20 \%$ of genes that exhibited MB subgroup-specific expression contained a pdCR, which suggests that this pattern plays an important regulatory role in distinct tumourspecific transcriptomes. Large, megabase-scale blocks of reduced DNA methylation (partially methylated domains, PMDs) represented another pattern of differential methylation in $\mathrm{MB}^{75}$. PMDs are a prominent feature in many cancer types and coincide with nuclear lamina-associated domains and other heterochromatic regions ${ }^{91-93}$. In $\mathrm{MB}$, PMDs are primarily detected in the WNT and Group 3 subgroups and can cover up to one-third of the genome, often in a subgroup-specific manner.

The integration of MB RNA-seq and enhancer ChIPseq (namely, H3K27ac) datasets has further illuminated the mechanisms of gene regulation ${ }^{94}$. Enhancer ChIPseq data generated for 28 primary MBs and $3 \mathrm{MB}$ cell lines enabled annotation of the active cis-regulatory landscape across MB subgroups. In total, nearly 80,000 enhancers were inferred, $25 \%$ of which had not previously been annotated by the Encyclopedia of DNA Elements (ENCODE) ${ }^{95,96}$ or the Roadmap Epigenomics Consortium $^{97}$. Computationally linking highly active, subgroup-specific enhancers, or 'super-enhancers', to putative target genes revealed new insights into the gene regulatory networks underlying $\mathrm{MB}$ subgroup biology and identity. Known cancer-associated genes were identified as prominent super-enhancer targets, including $A L K$ (encoding the ALK receptor tyrosine kinase, RTK) in WNT MB; GLI2, SMO and neurotrophic RTK 3 (NTRK3) in SHH $\mathrm{MB}$; the transcriptional regulators $L M O 1$ and $L M O 2$, as well as $M Y C$ in Group $3 \mathrm{MB}$; and the transcription factors ETV4 and PAX5 in Group $4 \mathrm{MB}$, among others. Differential analysis of enhancers and their inferred gene targets identified aberrant transforming growth factor- $\beta$ (TGF $\beta$ ) signalling activity that was specific to Group 3, substantiating prior genomic-based evidence implicating oncogenic TGF $\beta$ signalling in a subset of tumours?

\section{Proteomic analyses}

During the past decade, tremendous technical progress has been made towards sensitive detection and accurate quantification of the proteome ${ }^{98}$, and recent studies have begun to explore the proteomic landscape of MB. Quantitative mass spectrometry (MS)-based proteomics of primary human MBs has predominantly confirmed the classification of $\mathrm{MB}$ into consensus subgroups in three independent cohorts ${ }^{99-101}$. The proteome also revealed notable substructure within both the $\mathrm{SHH}$ (designated as SHH-a and SHH-b) and Group 3 (designated as Groups 3a and 3b) subgroups. These observations were not immediately supported by companion DNA methylation or transcriptomic datasets, indicating a potential role for post-transcriptional mechanisms in the pathobiology of SHH MB.

Concordance between mRNA transcripts and protein abundance is known to vary between species and cell types, with only $\sim 40 \%$ of protein variance explained by variance in mRNA abundance ${ }^{102}$. Group 3 and Group 4 MBs exhibit the lowest correlation between mRNA and protein expression, emphasizing the potential role of post-transcriptional mechanisms in their underlying biology $y^{100,101}$. At the proteomic level, Group $3 \mathrm{MB}$ exhibited elevated expression of subunits of eukaryotic initiation factor (eIF: EIF2s, EIF3s, EIF4Gs and EIF4As) ${ }^{100}$, a complex implicated in the initiation of protein synthesis in eukaryotes. Supporting this finding, pharmacological inhibition of formation of the eIE4F complex reduced the viability of cell lines derived from Group 3 $\mathrm{MBs}$ in vitro ${ }^{101}$. Interestingly, discrepancies between mRNA and protein expression revealed activation of RTK signalling in Group 4 through aberrant expression of ERBB4 and the phosphorylated form of the tyrosine-protein kinase SRC (FIG. 3). In utero (embryonic day 13.5) electroporation-mediated overexpression of activated SRC in combination with dominant-negative p53 in the developing mouse cerebellum induced Group 4-like $\mathrm{MB}^{100}$, functionally substantiating observations gleaned through proteomics. While the mechanisms responsible for the mRNA- and protein-level discrepancies seen in Group 3 and Group 4 MBs are likely due to a multitude of factors, translational effects mediated through MYC or MYCN are suspected to play a role ron,103,104. $_{\text {. }}$.

Phosphoproteomics can indicate protein kinase activity and potential opportunities for therapeutic intervention through the administration of pharmacological inhibitors. Bioinformatic analyses of MB phosphoproteomic data predicted the activation of several prominent kinases, including glycogen synthase kinase $3 \beta$ (GSK3 $\beta$; Group 4 and SHH-b), PRKDC (also known as DNA-PKcs; Group 3 and WNT), cyclin-dependent kinase 5 (CDK5; Group 4) and CDC-like kinase 1 (CLK1; Group 3) and casein kinase 2 (CK2; Group 3). Also, kinome analyses using high-throughput peptide phosphorylation profiling revealed two distinct proteinsignalling signatures in MB: MYC-like protein signalling, observed in the majority of $\mathrm{SHH}$ and Group $3 \mathrm{MBs}$, and protein-signalling profile 2 , characterized by DNA damage response and apoptotic and neuronal signalling in the majority of Group $4 \mathrm{MBs}\left(\mathrm{REF}^{104}{ }^{104}\right.$. Hence, these findings reinforce the idea that protein activity might reveal unifying and/or distinct tumour biologies among MB subgroups. Also, several post-translational modifications (PTMs) of key players in MB have been 


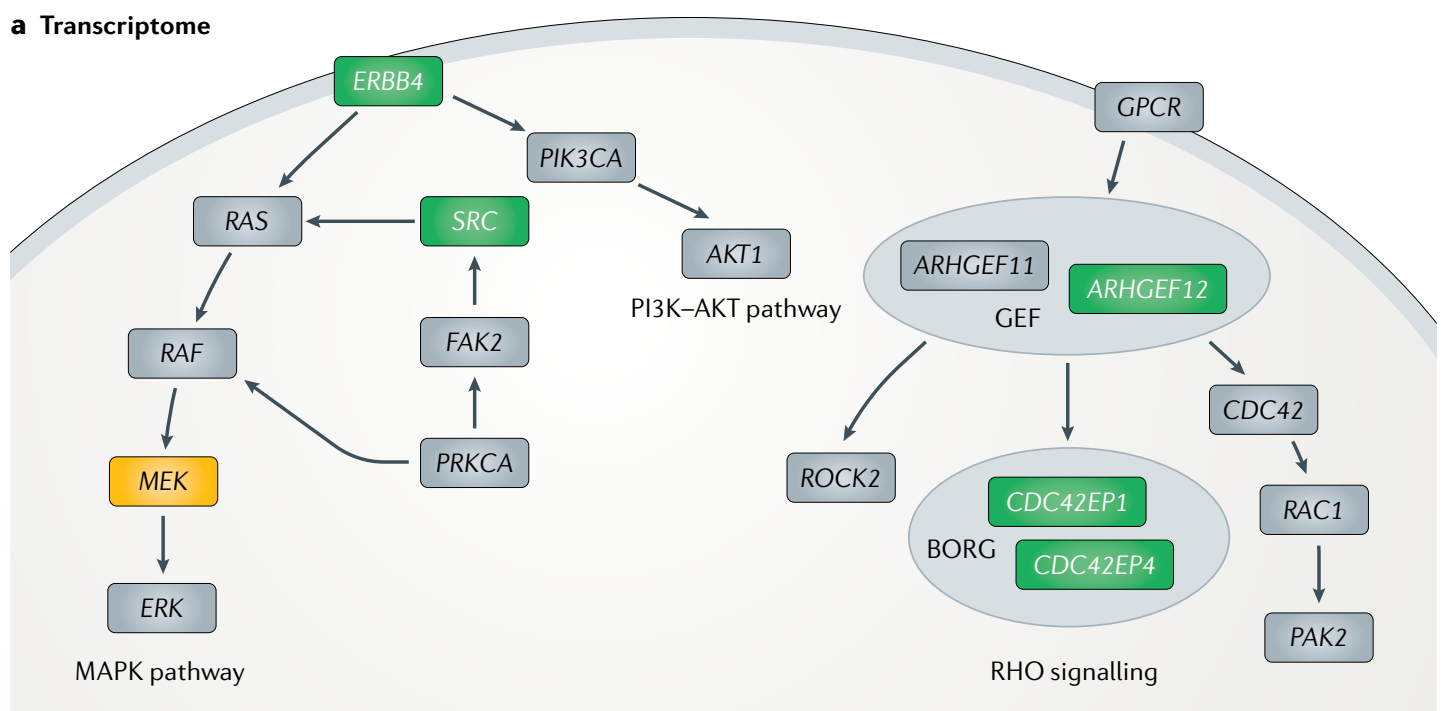

b Proteome and phosphoproteome

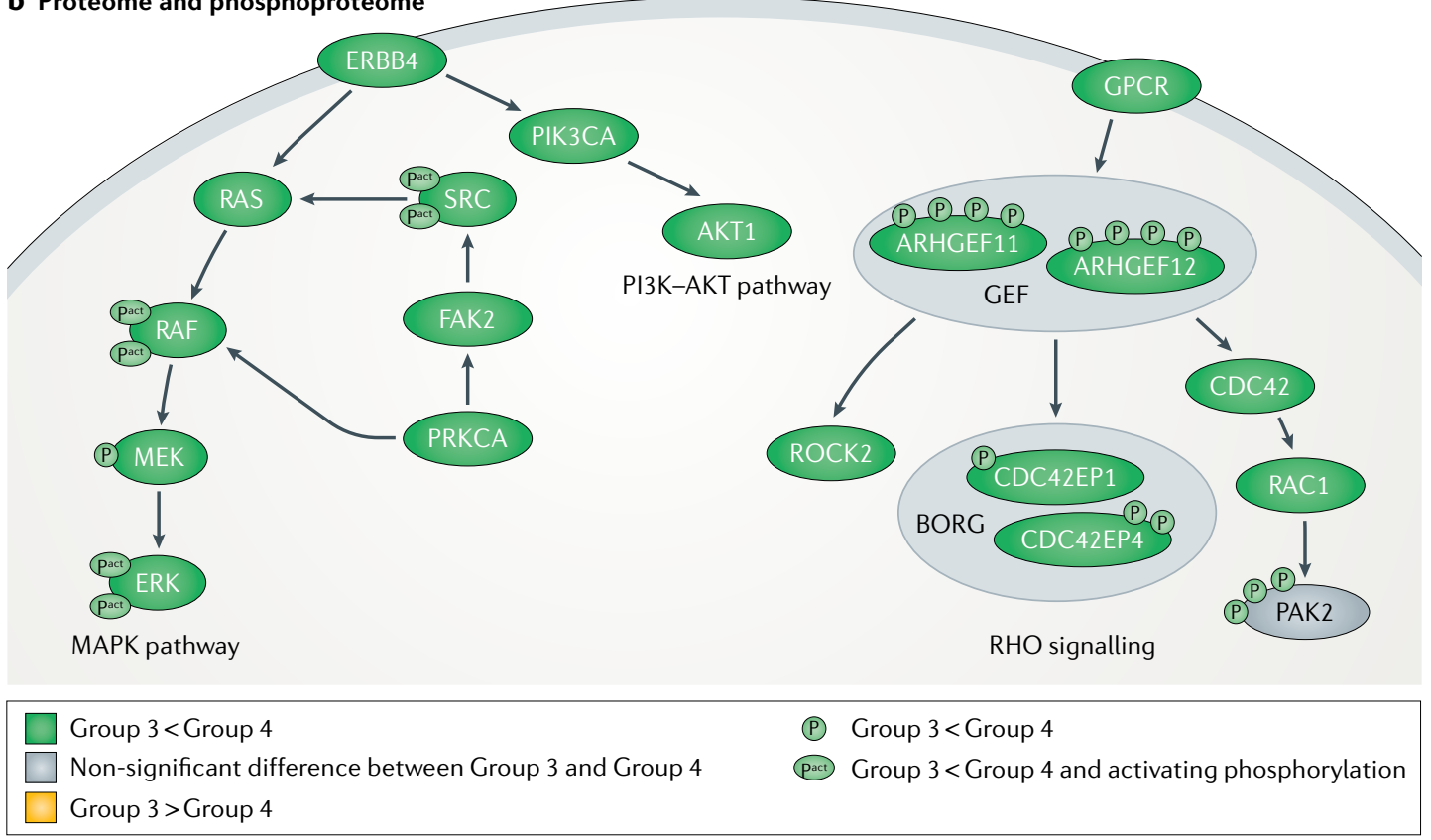

Fig. 3 | Aberrant signalling pathways implicated in Group 3 and Group 4 MB by proteomics. Colours indicate differential expression of mRNA (part a; $P<0.05$, $t$-test) or protein and phosphoprotein abundances (part $\mathbf{b}$ ) between Group 3 and Group 4 medulloblastomas (MBs), as reported in the Forget et al. study ${ }^{100}$. Phosphorylation is indicated as a circled $\mathrm{P}$, and $\mathrm{Pact}$ indicates that the phosphorylation event has previously been shown to activate the protein. ARHGEF11, RHO guanine nucleotide exchange factor 11; BORG, binder of RHO GTPases family; CDC42EP1, CDC42 effector protein 1; FAK2, focal adhesion kinase 2; GEF, guanine nucleotide exchange factor; GPCR, G protein-coupled receptor; PAK2, p21 activated kinase 2; PIK3CA, PI3K catalytic subunit $\alpha$; PRKCA, protein kinase $\mathrm{C} \alpha$; ROCK2, RHO-associated protein kinase 2.

identified. For instance, in SHH MB, deacetylation of GLI1 and GLI2 proteins induces their transcriptional activity ${ }^{105}$, while phosphorylation of the transcription factor ATOH1 by Janus kinase 2 (JAK2) controls tumour growth ${ }^{106}$. Besides phosphorylation and acetylation, a myriad of other PTMs, including ubiquitylation, methylation and others, have yet to be investigated in MB. The field of proteomics is rapidly advancing, and, along with further technological and bioinformatic breakthroughs, a deeper characterization of $\mathrm{MB}$ subgroup biology will undoubtedly follow.

\section{Intratumoural heterogeneity}

Phenotypic heterogeneity of individual cells within tumours has been a longstanding interest in MB research. Following similar discoveries in other solid tumours ${ }^{107}$ and in leukaemia ${ }^{108,109}$, Peter Dirks and colleagues first reported the discovery of a stem-like tumour cell population in samples from patients with MB characterized by the neural stem cell surface marker CD133 (positive for $6-21 \%$ of cells) $)^{110,111}$. These cells show a marked capacity for proliferation, self-renewal and neuronal differentiation, both in vitro by neurosphere 


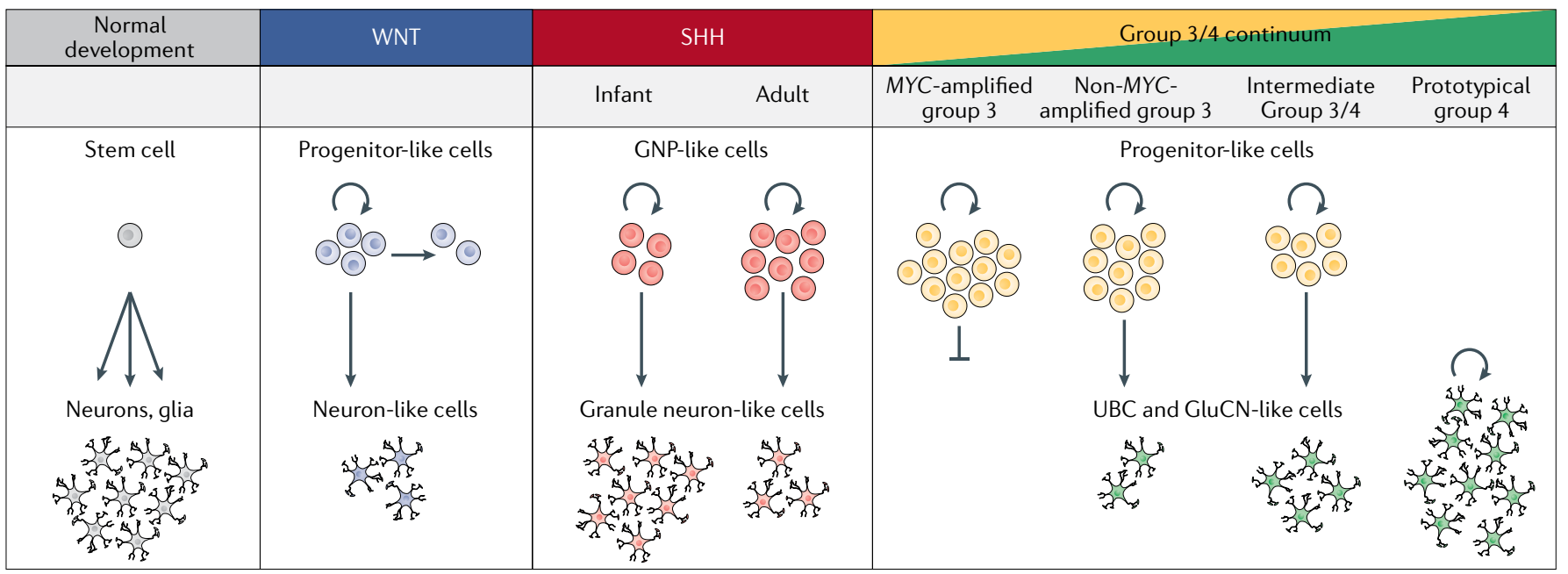

Single-cell RNA sequencing Emerging technology that enables unsupervised characterization of transcriptional profiles in individual cells of healthy and diseased tissues. The throughput of this technology has steadily increased over recent years, now enabling profiling of tens of thousands of individual cells in a single experiment

Granule neuron progenitor (GNP). A progenitor cell type that gives rise to granule cells, the most common type of neuron in the mature cerebellum; the presumed developmental origin of Sonic hedgehog medulloblastoma.

Unipolar brush cells (UBCs). Rare glutamatergic interneurons found in the cerebellar cortex and in the dorsal cochlear nucleus. Recent studies have identified transcriptional similarities between UBCs in mouse and human Group 4 medulloblastoma

\section{Glutamatergic cerebellar} nuclei

(GluCN). Also referred to as deep cerebellar nuclei. Cells that function (along with GABAergic interneurons) as the main output centres of the cerebellum

Fig. 4 | MB subgroup cellular hierarchies deduced from single-cell RNA sequencing. Illustration of malignant cell types inferred in medulloblastoma (MB) subgroups ${ }^{121,122}$. WNT MB comprises undifferentiated progenitor-like populations and more differentiated neuron-like cells. The undifferentiated, cycling cells in Sonic hedgehog (SHH) MB resemble granule neuron progenitor (GNP) cells, whereas differentiated cells resemble granule neurons. The frequency of these cell populations varies with age-associated subtypes, with more differentiated cells in infant patients and more GNP-like cells in adults. Group 3 and Group 4 MBs comprise undifferentiated progenitor-like cells and more differentiated types of neurons that share expression of marker genes with glutamatergic cerebellar nuclei (GluCN) and unipolar brush cells (UBCs). A continuum of frequencies of these cell types is observed, with Group 3 tumours being fully or mostly undifferentiated, Group 4 tumours being predominantly differentiated and some intermediate tumours being located in between. These transcriptional states are superimposed on genetic alterations that are associated with molecular subtypes.

culture and in vivo by mouse xenotransplantation. In mice, as few as $1,000 \mathrm{CD} 133^{+}$cells were sufficient to initiate tumours that phenotypically resembled the original tumour, whereas 50,000 CD133- cells failed to establish tumours. CD133 is most highly expressed in Group 3 $\mathrm{MB}^{112}$, but it does not mark tumour-propagating cells in SHH-MB GEM models ${ }^{113,114}$. Instead, the neural stem cell surface antigen CD15 was found to enrich for tumour-propagating cells in this subgroup. Further analysis of the $P t c h 1^{+/}$SHH-MB GEM model identified a rare, quiescent $\mathrm{SOX}_{2}{ }^{+}$cell population that gave rise to rapidly cycling progenitors ${ }^{115}$

In recent years, single-cell transcriptome sequencing (that is, single-cell RNA sequencing; scRNA-seq) has emerged as a powerful method to decipher cellular states in healthy and diseased tissues in an unbiased way, as exemplified in adult and paediatric gliomas ${ }^{116-120}$. Recently, two independent studies applied scRNA-seq to cohorts of primary MBs, showing that MB displays subgroup-specific transcriptional heterogeneity at the single-cell level ${ }^{121,122}$. Analysing eight patients from either the SHH, Group 3 or Group 4 subgroup, Vladoiu and colleagues demonstrated mixed populations of cells with divergent differentiation along cerebellar neuronal lineages ${ }^{121}$. Analysing 25 patients across all molecular subgroups, we identified subgroup-specific undifferentiated and differentiated neuronal-like malignant populations ${ }^{122}$ (FIG. 4). Both studies confirmed the resemblance of SHH tumours to cerebellar granule neuron progenitor (GNP) cells, in agreement with earlier experimental evidence $^{13,123}$ (FIG. 4; BOX 1). Interestingly, adult SHH-MB tumours showed a higher fraction of undifferentiated GNPs than did infant tumours, which might be linked to the divergent biologies of these groups ${ }^{122}$ (FIG. 4).
Combined analysis of Group 3 and Group 4 tumours revealed a related developmental trajectory, from primitive and progenitor-like to more mature, neuronal-like cells, the relative proportions of which distinguished the subgroups $^{122}$ (FIG. 4). MYC-amplified Group 3 MBs only comprised undifferentiated progenitor-like cells and did not show any capacity to differentiate ${ }^{122}$. Most other Group 3 MBs showed a small degree of neuronal differentiation ( $<10 \%$ of cells), indicating that tumour cells maintained the capacity to differentiate. Interestingly, a subset of tumours characterized as 'intermediate' cases by DNA methylation-based classification exhibited both undifferentiated and differentiated populations in varying proportions ${ }^{122}$, providing an explanation for the known challenges associated with confident classification of some Group 3 and Group $4 \mathrm{MB}$ cases to either subgroup by bulk molecular profiling (FIG. 4). Prototypic Group 4 MBs almost exclusively comprised more differentiated neuronal-like cells resembling unipolar brush cells $(\mathrm{UBCs})^{121,122}$ and glutamatergic cerebellar nuclei (GluCN) $)^{122}$ (FIG. 4; see BOX 1).

Recent parallel gene expression profiling and exome sequencing of 47 multiregional biopsies from eight patients demonstrated that $\mathrm{MB}$ is characterized by spatially homogeneous transcriptomes, in which biopsies from the same patient were more similar to each other than to those of other patients ${ }^{124}$. This was contrary to findings in glioblastoma, in which different biopsies from the same patient were classified as different transcriptional subtypes ${ }^{124}$. However, the same study reported high levels of genetic heterogeneity in $\mathrm{MB}$ at the level of SCNAs and somatic mutations. As the affected genes included therapeutic targets, these observations put into question whether single biopsies 
are truly representative of the entire tumour and whether monotherapies can effectively treat $\mathrm{MB}$.

\section{Insights into relapse and metastasis}

Since $\mathrm{MB}$ relapse and metastases remain the most significant morbidity factors influencing patient outcomes ${ }^{125}$, an improved understanding of the molecular events driving treatment resistance and recurrence represents a major priority for the field. To date, comparative

\section{Box 1 | Cellular origins of MB subgroups}

Because medulloblastoma (MB) subgroups are enriched for specific genetic alterations and exhibit different epigenetic and transcriptional profiles, it has long been suspected that they arise from distinct cellular populations or developmental lineages ${ }^{143}$. The figure depicts an embryonic mouse cerebellum (estimated development at $\sim 13.5$ days) highlighting cellular populations proposed to be developmentally linked to specific MB subgroups. Mouse models of the WNT and Sonic hedgehog (SHH) subgroups have substantiated lower rhombic lip $(\mathrm{LRL})$ progenitors $\left(\mathrm{BLBP}^{+}\right)$and granule neuron progenitor (GNP) populations ( $\mathrm{ATOH} 1^{+}$), respectively, as probable cells of origin for these subgroups $^{144}$. Multiple orthotopic, transgenic and somatic gene transfer mouse models have demonstrated that a variety of stem/progenitor cell populations can be effectively transformed in order to replicate the molecular and phenotypic features of MYCN-driven $\mathrm{SHH}_{\mathrm{MB}}{ }^{145}$ or MYCN- or MYC-driven Group $3 \mathrm{MB}^{146-149}$. Mapping the MB enhancer landscape enabled inferences as to the master transcription factors (TFs) governing subgroup-specific tumour biology. LMX1A, EOMES and LHX2 were predicted to function as master regulators of Group $4 \mathrm{MB}^{94}$. Developmentally, these master TFs regulate lineage specification for restricted glutamatergic progenitor cell populations born out of the cerebellar upper rhombic lip (URL) during cerebellar morphogenesis ${ }^{150,151}$, including glutamatergic cerebellar nuclei (GluCN; also known as deep cerebellar nuclei) and unipolar brush cells (UBCs). Since LMX1A, EOMES and LHX2 were inferred to be highly specific super-enhancer-regulated candidate master TFs in Group 4 MB, the aforementioned glutamatergic populations expressing these markers were proposed as the putative lineages of origin for this $\mathrm{MB}$ subgroup ${ }^{94}$. More recently, comparative crossspecies transcriptomic analyses have complemented these observations, using singlecell transcriptional profiles of the developing mouse cerebellum as a reference for mapping MB subgroup origins ${ }^{121,122}$. Granule neuron lineage populations were highly correlated with SHH MBs, reinforcing the expansive literature implicating GNPs as their developmental origin. Interestingly, GluCN and UBCs were both shown to be highly transcriptionally similar to Group $4 \mathrm{MBs}$, suggesting that these populations could represent bona fide cells of origin for this subgroup of patients. Early embryonic Nestin ${ }^{+}$ progenitor cells were suggested as being highly correlated with Group $3 \mathrm{MB}^{121}$; however, these results were not supported in the companion study ${ }^{122}$. Deeper molecular analyses of these novel candidate populations and functional studies that mimic the relevant candidate driver alterations in the correct lineage at the correct developmental stage are ongoing and will be required in order to confirm these initial findings.

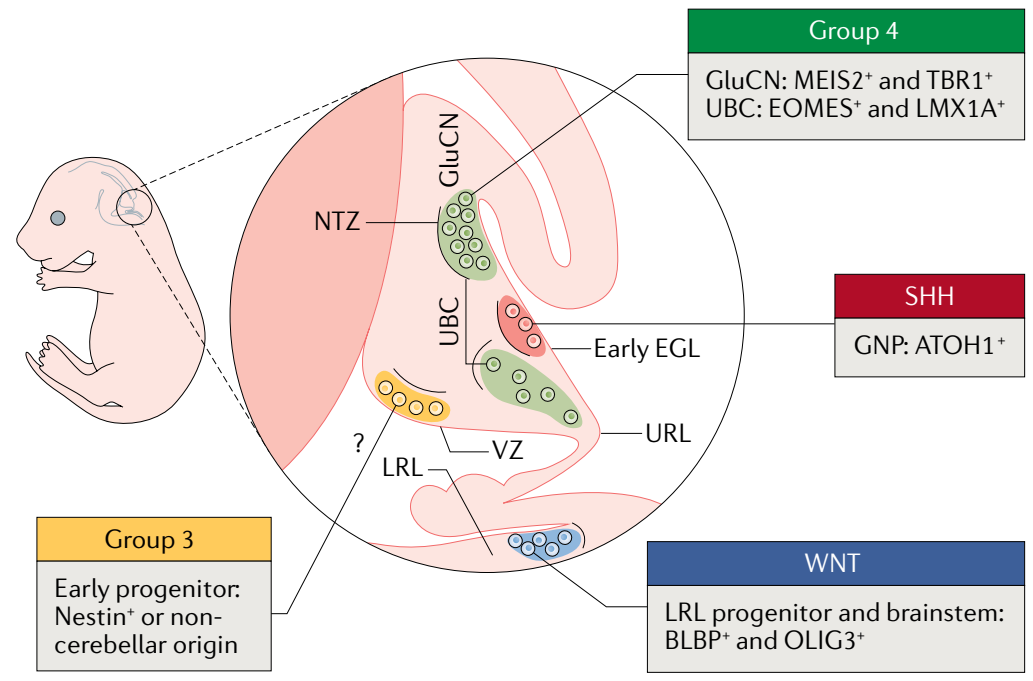

EGL, external granule layer; NTZ, nuclear transitory zone; VZ, ventricular zone. studies of primary versus relapsed disease have been exceedingly limited and restricted to relatively modest cohorts. Application of the NanoString-based molecular classification assay confirmed that MB subgroup status is conserved between primary and relapsed disease, reinforcing the concept that the individual MB subgroups constitute distinct diseases ${ }^{126}$. A complementary subgrouping approach later independently confirmed this observation ${ }^{127}$. The specific emergence of MYC or $M Y C N$ amplifications and p53 pathway defects (namely TP53 mutation and CDKN2A deletion) at relapse was also reported ${ }^{127}$. An elegant SHH-MB GEM model made using the Sleeping Beauty transposon system $\left(\right.$ Ptch $^{+/-}$, Math1-SB11, T2Onc or T2Onc2) functionally substantiated the genetic divergence of primary versus recurrent tumours ${ }^{128}$. In the same study, genomic characterization of a cohort of 36 primary and relapsed tumours from patients with $\mathrm{MB}$ indicated that recurrent tumours harboured a profound increase in mutational burden and SVs. More extensive efforts are ongoing that are sufficiently powered to build on these studies and to provide further understanding of the molecular basis of $\mathrm{MB}$ treatment failure and relapse.

MB metastasis usually occurs via leptomeningeal dissemination (LMD) that remains confined to the CNS and spinal cord ${ }^{125}$. Approximately one-third of all patients with $\mathrm{MB}$ have metastatic disease at diagnosis, with patterns and frequencies that vary considerably according to molecular subgroup ${ }^{129}$. Patients who fail conventional therapy and/or who relapse with metastatic disease share a universally dismal prognosis, with nearly all patients succumbing to their refractory disease. Garzia and colleagues inferred the presence of circulating tumour cells (CTCs) on the basis of careful analysis of specific mutations in simultaneously collected patient-matched primary $\mathrm{MB}$, leptomeningeal metastatic $\mathrm{MB}$ and peripheral blood ${ }^{130}$. These mutations were clonal in the metastasis, clonal or subclonal in the primary tumour, and present at very low fractions in the peripheral blood. In three of six peripheral blood samples, exceedingly rare $\mathrm{CD} 56^{+} / \mathrm{CD} 45^{-}$morphologically abnormal cells were identified, further supporting the presence of rare CTCs in the blood of some patients with MB. These molecular and cellular observations derived from patient samples were strengthened by a series of innovative mouse-modelling experiments, including the implementation of a parabiosis model that established the haematogenous spread of implanted MB cells between surgically connected donor (implanted mouse) and recipient (sibling mouse) animals. Comparison of gene expression profiles for a limited set of patientmatched primary and metastatic MB samples in the same study identified overexpression of the chemokine CCL2 in the metastatic compartment, which was further substantiated through additional molecular and functional analyses, suggesting that aberrant CCL2 signalling may be an important mediator of LMD in MB.

\section{Emerging clinical implications}

Risk stratification. There has been a cautious yet steady transition of discoveries made in the research arena into the clinic. Indeed, clinical protocols for patients with 
Sleeping Beauty transposon system

A synthetic DNA transposon system used for random mutagenesis screening and in a recent Sonic hedgehogmedulloblastoma mouse model. Genes affected by genomic insertion of the transposon can be identified through sequencing.

Bone age

Degree of skeletal maturity an important parameter for determining the clinical use of SMO inhibitors in patients with Sonic hedgehog medulloblastoma. Prolonged exposure to the targeted inhibitor vismodegib has been associated with growth defects in children that have not reached skeletal maturity.

Patient-derived xenograft (PDX). A model of cancer in which tumour cells from a patient are implanted and maintained in a non-human carrier, most commonly immunodeficient or humanized laboratory mice. PDX models are thought to resemble patient tumours more closely than cell cultures do.
MB have begun to implement molecular subgroupinformed strategies for treatment stratification. SJMB12 (NCT01878617 (REF. $\left.{ }^{131}\right)$ ), an active trial for newly diagnosed patients with $\mathrm{MB}$, includes separate treatment arms for patients in the WNT, SHH and non-WNT/ non-SHH (that is, Group 3 and Group 4) subgroups. According to this protocol, patients with clinically standard-risk WNT MB (non-metastatic with near total surgical tumour resection) receive reduced CSI ( $15 \mathrm{~Gy}$ as opposed to the standard dose of $23.4 \mathrm{~Gy}$ administered to patients with standard-risk $\mathrm{MB}$ ), owing to the highly favourable outcomes that have been consistently reported for WNT MB since $2005\left(\right.$ REFS $\left.^{33,34}\right)$. Skeletally mature (that is, females with a bone age $\geq 15$ years or males with a bone age $\geq 17$ years) patients with SHH MB who are enrolled in SJMB12 receive the SMO inhibitor vismodegib on top of standard-of-care chemotherapy and CSI. In contrast, patients with non-WNT/non-SHH forms of $\mathrm{MB}$ are further stratified into standard- and high-risk treatment arms based on a combination of clinical- and molecular-risk factors, including the extent of resection, metastatic status and MYC amplification status. A trial for newly diagnosed patients that is ongoing in Europe, International Society of Paediatric Oncology PNET 5 (NCT02066220 (REF. ${ }^{132}$ )), is likewise stratifying patients with WNT MB in a low-risk treatment arm and administering reduced-dose CSI (18 Gy) compared with the patients in other MB subgroups, who are treated according to standard of care; a similar trial for patients with WNT MB by the Children's Oncology Group (ACNS1422; NCT02724579 (REF. ${ }^{133}$ )) is ongoing in North America. Despite these examples of molecularly informed risk stratification in current MB trials, the clinical arena still lags behind the pace of retrospective research studies. Recent molecular studies conducted on both large retrospective and trial cohorts have provided an increased rationale for further molecularly driven stratification in future protocols, especially within specific MB subgroups ${ }^{29,31,35,44,134}$. However, due to the reasonably high cure rates for all patients with MB ( 70-75\%), the way forward must continue to be methodical. With children's lives at stake, rather than leaping into untested waters, trials need to simultaneously build on past successes, chip away at lessening the morbidities of current therapy and improve cure rates for the patient population who consistently fail to benefit from current therapies. The answer lies in using the new molecular understandings to improve risk stratification, such that judicious dose reductions can be applied to the lowest-risk groups while promising experimental therapy can be given to the highest-risk patients. In this way, reductions in long-term treatment-related morbidities are prioritized for patients who are surviving, and the chance of survival is increased for patients who are currently dying of the disease.

Genetic predisposition. Hereditary genetic predisposition to $\mathrm{MB}$ remains an underappreciated clinical challenge. Recent data collected on large retrospective $\mathrm{MB}$ cohorts suggest that, especially in the case of SHH MB, the proportion of patients with an underlying cancer predisposition might approach $25 \%$ or even higher ${ }^{36}$. In patients with WNT MB, the proportion appears to be in the range of 5-10\%. At this stage, these estimates only account for known cancer predisposition genes, and efforts to systematically identify other pathogenic events in the germ line of patients with MB are currently ongoing. American Association for Cancer Research (AACR) guidelines recommend genetic testing and counselling for paediatric tumour types that frequently occur in the context of genetic predisposition (where a threshold of $\geq 10 \%$ of a given cancer's incidence is attributed to pathogenic germline mutations) ${ }^{135}$. The prevalence of pathogenic germline events in known cancer predisposition genes qualifies $\mathrm{SHH} \mathrm{MB}$, and potentially WNT MB, for a general recommendation to offer genetic testing and counselling prior to adjuvant therapy. This infrastructure, however, is currently not in place in the majority of treatment centres across the world. The largest $\mathrm{MB}$ predisposition study to date ${ }^{36}$ identified $A P C$ germline mutations in patients with CTNNB1 mutation-negative WNT MB, as well as significant enrichment of TP53 (Li-Fraumeni syndrome), SUFU, PTCH1 (Gorlin syndrome), BRCA2 and PALB2 pathogenic germline variants in SHH MB. These genes can be tested together by clinical-grade whole-exome or panel sequencing, but also successively as single-gene tests based on their age associations and, in some cases, a family history. AACR surveillance guidelines are available for APC, TP53, SUFU and PTCH1, whereas it remains to be determined how to appropriately manage patients with damaging heterozygous germline $\mathrm{MB}$ variants in $B R C A 2$ and $P A L B 2$. As this considerable proportion of patients with hereditary MB disease and their families require special clinical attention, including potential treatment modifications, family testing and surveillance, this challenge has to be tackled systematically but expeditiously.

Therapeutic targets. Arguably, it was the discovery of small molecule Hedgehog pathway antagonists (that is, SMO inhibitors) and their preclinical potency against Ptch ${ }^{+/-}$GEM models ${ }^{136}$ that sparked the need to molecularly identify patients with MB who would benefit from these inhibitors. However, what emerged was molecularly far more complex than had first been envisioned. $\mathrm{SHH}$ pathway gene alterations in $\mathrm{SHH} \mathrm{MB}$ differ according to patient age at diagnosis and subtype $\mathrm{e}^{29,30,38,44}$, collectively accounting for the variable responses to SMO inhibitors. PTCH1 mutations (both germline and somatic) are the most common ones but are present in less than half of all patients with SHH MB, and mostly within infants and adults ${ }^{30,38,44}$. Germline and somatic SUFU mutations are largely restricted to infant $\mathrm{SHH}$ MBs, whereas activating somatic SMO mutations are enriched in adult patients with $\mathrm{SHH} \mathrm{MB}{ }^{30,38,44}$. Similarly, germline and somatic TP53 mutations, predominantly coincident with GLI2 and MYCN amplifications, are found exclusively in children between the ages of 8 and 17 years $^{29,30,38}$. These observations are of direct clinical relevance when considering the treatment of SHH MB with SMO inhibitors. The Ptch $1^{+/-}$GEM model ${ }^{136}$ and a patient-derived xenograft (PDX) model from a patient with $\mathrm{SHH} \mathrm{MB}^{38}$ harbouring a $\mathrm{PTCH} 1$ mutation were sensitive to SMO inhibitors (HhAntag, vismodegib or sonidegib), whereas a GEM model lacking $S u f u^{137}$ or 
Blood-brain barrier

A semipermeable border formed by endothelial cells lining the cerebral

microvasculature that separates the brain from the circulating blood and protects the brain from fluctuations in plasma composition and from circulating agents such as neurotransmitters and pathogens. The blood-brain barrier also presents a challenge for drug delivery when treating brain tumours.

Blood-tumour barrier Tumour-associated compromise of the bloodbrain barrier, resulting in a highly heterogeneous vasculature characterized by non-uniform permeability to small and large molecules.
PDX harbouring TP53 mutation and MYCN amplification exhibited primary resistance to these inhibitors ${ }^{38}$. These preclinical findings were corroborated in an earlyphase clinical trial on patients with relapsed $\mathrm{SHH} \mathrm{MB}$ who were treated with vismodegib ${ }^{138}$. As was molecularly predicted, responders to SMO inhibition were more likely to have tumours that harboured a PTCH1 mutation, whereas no beneficial clinical responses were observed in patients harbouring TP53 or SUFU mutations and concurrent MYCN and GLI2 amplifications. Moreover, another significant concern that has restricted the clinical use of SMO inhibitors is the emergence of permanent growth plate fusions in the long bones of small children ${ }^{139}$, a morbidity that was predicted in early preclinical studies of young mice ${ }^{140}$. Given this, the current clinical strategy has shifted to skeletally mature patients with upstream (that is, $P T C H 1$ or $S M O$ ) $\mathrm{SHH}$ pathway mutations. While this is an admittedly small population, this approach attempts to first define the benefit of SMO inhibitors in a targeted population, prior to exposing a more vulnerable population to the inhibitors and their morbidities. Collectively, these findings demonstrate the importance of performing thorough preclinical and molecular testing to identify the appropriate patient populations as candidates for molecularly targeted therapy.

Additional translational implications relevant to other MB subgroups have also emerged. For instance, the oncogenic activity of GFI1 in MB is promoted through direct interaction with KDM1A (also known as LSD1) ${ }^{141}$, a histone lysine demethylase associated with transcriptional repression. Treatment of MBs driven by MYC and GFI1 with LSD1 inhibitors attenuated the malignant phenotype in vitro and in vivo in a flank PDX model. No treatment effect was observed when treating the same tumours in an orthotopic setting, demonstrating that this model might serve as a preclinical testing vehicle for blood-brain barrier or blood-tumour barrier penetration. Taken together, these results provide preclinical support for treating patients with GFI1 (or GFI1B)-driven MB with LSD1 inhibitors, if a brain-penetrant LSD1 inhibitor becomes available.

With the identification of aberrant TGF $\beta$ signalling in Group $3 \mathrm{MB}$, and RTK signalling in Group $4 \mathrm{MB}$, new opportunities for preclinical testing of inhibitors of these pathways have likewise emerged ${ }^{100,142}$. Additional studies evaluating the efficacy of such agents in treating accurate preclinical MB models will be necessary before their translation to the clinic.

\section{Conclusion and future outlook}

Through continued multi-omic analyses conducted on unprecedented patient cohorts, $\mathrm{MB}$ now represents one of the most extensively characterized cancer entities. Deep understanding of its molecular substructure and the assignment of known and novel driver gene alterations to specific disease subtypes have created a more refined understanding of MB tumour biology. This knowledge will enable the development of better models that more accurately recapitulate human disease. Moreover, these advances pave the way for the critical functional studies that will be required in order to determine the mechanistic contribution of newly discovered genes and molecular complexes to $\mathrm{MB}$ pathogenesis, in the appropriate cellular context. A continued transition from bulk tumour profiling to more detailed analyses of intratumoural heterogeneity in single cells is expected, especially as methods for analysing genetic alterations and the epigenome, transcriptome and proteome at single-cell resolution continue to evolve and become attainable on archival clinical samples. These studies should help further resolve the recently described molecular subtypes within subgroups and elucidate the molecular and cellular basis of MB recurrence and metastasis.

Clinically, the separation of MB along molecular lines into subgroups, and now into subtypes within subgroups, generates concern among many treating physicians that the number of divisions far outpaces our ability to give subtype-specific care. However, while this argument against a complicated classification system is understandable, given that the majority of subtypespecific findings are not immediately targetable by individual medicines, it ignores the clinical benefit of improved risk stratification. Despite reasonably high cure rates, in the range of $70-75 \%$ for all patients with $\mathrm{MB}$, the cost of current therapy that combines surgery, CSI and chemotherapy remains unacceptably high. Molecular subgrouping has already demonstrated pronounced differences in survival, and this has afforded the opportunity to trial judicious reductions in therapy to the lower-risk WNT subgroup, while maintaining and optimizing intensive therapy for the high-risk patients with Group $3 \mathrm{MB}$. Moreover, the more precise and more well defined the subtypes become, the better the opportunity to hone this risk stratification approach. For example, the new subtyping of very young children with $\mathrm{MB}$ is anticipated to rapidly lead to new trials, as the toxicities of CSI and chemotherapy remain unacceptably high in this most vulnerable population ${ }^{44}$. Furthermore, if promising targeted therapies, such as SMO inhibitors, LSD1 inhibitors and RTK inhibitors, are to be successful, then it is imperative that these agents be given to the appropriate populations. Even though the end of further evolution of MB molecular sub-classification may be in sight, the impact of these molecularly informed advances on therapy is only just beginning.

Published online 9 December 2019
1. Ostrom, Q. T. et al. CBTRUS Statistical Report: primary brain and other central nervous system tumors diagnosed in the United States in 2011-2015. Neuro-Oncology 20, iv1-iv86 (2018).

2. Curtin, S. C., Minino, A. M. \& Anderson, R. N Declines in cancer death rates among children and adolescents in the United States, 1999-2014. NCHS Data Brief, 1-8 (2016).
3. Ezzat, S. et al. Pediatric brain tumors in a low/middle income country: does it differ from that in developed world? J. Neurooncol. 126, 371-376 (2016).

4. Makino, K., Nakamura, H., Yano, S., Kuratsu, J. \& Kumamoto Brain Tumor Group. Population-based epidemiological study of primary intracranial tumors in childhood. Childs Nerv. Syst. 26, 1029-1034 (2010).
5. Taylor, M. D. et al. Molecular subgroups of medulloblastoma: the current consensus. Acta Neuropathol. 123, 465-472 (2012).

6. Northcott, P. A et al. Medulloblastomics: the end of the beginning. Nat. Rev. Cancer 12, 818-834 (2012).

7. Rausch, T. et al. Genome sequencing of pediatric medulloblastoma links catastrophic DNA 
rearrangements with TP53 mutations. Cell 148 59-71 (2012).

This first whole-genome sequencing study of MB identifies the association between TP53 mutations and chromothripsis in SHH MB

8. Pugh, T. J. et al. Medulloblastoma exome sequencing uncovers subtype-specific somatic mutations. Nature 488, 106-110 (2012)

9. Northcott, P. A. et al. Subgroup-specific structural variation across 1,000 medulloblastoma genomes. Nature 488, 49-56 (2012).

10. Jones, D. T. et al. Dissecting the genomic complexity underlying medulloblastoma. Nature 488, 100-105 (2012)

11. Northcott, P. A. et al. Medulloblastoma. Nat. Rev. Dis. Primers 5, 11 (2019).

12. Pomeroy, S. L. et al. Prediction of central nervous system embryonal tumour outcome based on gene expression. Nature 415, 436-442 (2002).

13. Lee, Y. et al. A molecular fingerprint for medulloblastoma. Cancer Res. 63, 5428-5437 (2003).

14. Cho, Y. J. et al. Integrative genomic analysis of medulloblastoma identifies a molecular subgroup that drives poor clinical outcome. J. Clin. Oncol. 29 1424-1430 (2011)

15. Kool, M. et al. Integrated genomics identifies five medulloblastoma subtypes with distinct genetic profiles, pathway signatures and clinicopathological features. PLOS ONE 3, e3088 (2008)

16. Northcott, P. A. et al. Medulloblastoma comprises four distinct molecular variants. J. Clin. Oncol. 29 , 1408-1414 (2011).

17. Thompson, M. C. et al. Genomics identifies medulloblastoma subgroups that are enriched for specific genetic alterations. J. Clin. Oncol. 24 1924-1931 (2006).

Cho et al. (2011), Kool et al. (2008), Northcott et al. (2011) and Thompson et al. (2006) report the first separation of MB into distinct molecular subgroups using array-based gene expression profiling and unsupervised clustering.

18. Louis, D. N. et al. The 2016 World Health Organization classification of tumors of the central nervous system: a summary. Acta Neuropathol. 131, 803-820 (2016)

19. Min, H. S., Lee, J. Y., Kim, S. K. \& Park, S. H. Genetic grouping of medulloblastomas by representative markers in pathologic diagnosis. Trans/ Oncol. 6, 265-272 (2013)

20. Ellison, D. W. et al. Medulloblastoma: clinicopathological correlates of SHH, WNT, and nonSHH/WNT molecular subgroups. Acta Neuropathol. 121, 381-396 (2011)

21. Northcott, P. A. et al. Rapid, reliable, and reproducible molecular sub-grouping of clinical medulloblastoma samples. Acta Neuropathol. 123, 615-626 (2012).

22. Leal, L. F. et al. Reproducibility of the NanoString 22-gene molecular subgroup assay for improved prognostic prediction of medulloblastoma. Neuropathology 38, 475-483 (2018).

23. Schwalbe, E. C. et al. Rapid diagnosis of medulloblastoma molecular subgroups. Clin. Cancer Res. 17, 1883-1894 (2011).

24. Capper, D. et al. DNA methylation-based classification of central nervous system tumours. Nature $\mathbf{5 5 5}$ 469-474 (2018).

25. Hovestadt, V. et al. Robust molecular subgrouping and copy-number profiling of medulloblastoma from small amounts of archival tumour material using highdensity DNA methylation arrays. Acta Neuropathol. 125, 913-916 (2013).

26. Schwalbe, E. C. et al. DNA methylation profiling of medulloblastoma allows robust subclassification and improved outcome prediction using formalin fixed biopsies. Acta Neuropathol. 125, 359-37 (2013).

Hovestadt et al. (2013) and Schwalbe et al. (2013) establish the use of genome-wide DNA methylation arrays for molecular subgrouping of MB.

27. Schwalbe, E. C. et al. Minimal methylation classifier (MIMIC): a novel method for derivation and rapid diagnostic detection of disease-associated DNA methylation signatures. Sci. Rep. 7, 13421 (2017).

28. Korshunov, A. et al. DNA-methylation profiling discloses significant advantages over NanoString method for molecular classification of medulloblastoma. Acta Neuropathol. 134, 965-967 (2017).

29. Cavalli, F. M. G. et al. Intertumoral heterogeneity within medulloblastoma subgroups. Cancer Cell 31 737-754.e6 (2017).
30. Northcott, P. A. et al. The whole-genome landscape of medulloblastoma subtypes. Nature 547, 311-317 (2017). This study has integrated and expanded earlier next-generation sequencing studies, for the most exhaustive genomic characterization of MB to date.

31. Schwalbe, E. C e et al. Novel molecular subgroups for clinical classification and outcome prediction in childhood medulloblastoma: a cohort study. Lancet Oncol. 18, 958-971 (2017).

Cavalli et al. (2017), Northcott et al. (2017) and Schwalbe et al. (2017) have described further subdivisions of molecular subgroups of MB into subtypes through molecular profiling of increasingly larger patient cohorts.

32. Kool, M. et al. Molecular subgroups of medulloblastoma: an international meta-analysis of transcriptome, genetic aberrations, and clinical data of WNT, SHH, Group 3, and Group 4 medulloblastomas. Acta Neuropathol. 123 , 473-484 (2012)

33. Gajjar, A. et al. Risk-adapted craniospinal radiotherapy followed by high-dose chemotherapy and stem-cell rescue in children with newly diagnosed medulloblastoma (St Jude Medulloblastoma-96): long-term results from a prospective, multicentre trial. Lancet Oncol. 7, 813-820 (2006).

34. Ellison, D. W. et al beta-Catenin status predicts a favorable outcome in childhood medulloblastoma: the United Kingdom Children's Cancer Study Group Brain Tumour Committee. J. Clin. Oncol. 23, 7951-7957 (2005).

35. Shih, D. J. et al. Cytogenetic prognostication within medulloblastoma subgroups. J. Clin. Oncol. 32 886-896 (2014)

36. Waszak, S. M. et al. Spectrum and prevalence of genetic predisposition in medulloblastoma: a retrospective genetic study and prospective validation in a clinical trial cohort. Lancet Oncol. 19, 785-798 (2018) This study describes germline predisposition to $\mathrm{MB}$, concluding that pathogenic germline mutation account for at least $6 \%$ of MB diagnoses.

37. Remke, M. et al. Adult medulloblastoma comprises three major molecular variants. J. Clin. Oncol. 29 , 2717-2723 (2011)

38. Kool, M. et al. Genome sequencing of $\mathrm{SHH}$ medulloblastoma predicts genotype-related response to smoothened inhibition. Cancer Cell 25, 393-405 (2014).

39. Northcott, P. A. et al. Pediatric and adult sonic hedgehog medulloblastomas are clinically and molecularly distinct. Acta Neuropathol. 122, 231-240 (2011).

40. Remke, M. et al. TERT promoter mutations are highly recurrent in SHH subgroup medulloblastoma. Acta Neuropathol. 126, 917-929 (2013).

41. Koelsche, C. et al. Distribution of TERT promoter mutations in pediatric and adult tumors of the nervous system. Acta Neuropathol. 126, 907-915 (2013).

42. Lindsey, J. C. et al. TERT promoter mutation and aberrant hypermethylation are associated with elevated expression in medulloblastoma and characterise the majority of non-infant $\mathrm{SHH}$ subgroup tumours. Acta Neuropathol. 127, 307-309 (2014).

43. Poschl, J. et al. Genomic and transcriptomic analyses match medulloblastoma mouse models to their human counterparts. Acta Neuropathol. 128, 123-136 (2014).

44. Robinson, G. W. et al. Risk-adapted therapy for young children with medulloblastoma (SJYC07): therapeutic and molecular outcomes from a multicentre, phase 2 trial. Lancet Oncol. 19, 768-784 (2018).

This study defines the molecular landscape of infant MB and integrates molecular findings with a prospective clinical trial cohort, concluding that infant SHH MB can be discriminated into two molecularly distinct subtypes with disparate survival outcomes

45. Wang, J., Garancher, A., Ramaswamy, V. \& Wechsler-Reya, R. J. Medulloblastoma: from molecular subgroups to molecular targeted therapies. Annu. Rev. Neurosci. 41, 207-232 (2018).

46. Ramaswamy, V. \& Taylor, M. D. Medulloblastoma: from myth to molecular. J. Clin. Oncol. 35 2355-2363 (2017).

47. Ramaswamy, V. et al. Risk stratification of childhood medulloblastoma in the molecular era: the current consensus. Acta Neuropathol. 131, 821-831 (2016).

48. Sharma, T. et al. Second-generation molecular subgrouping of medulloblastoma: an international meta-analysis of Group 3 and Group 4 subtypes. Acta Neuropathol. 138, 309-326 (2019).
49. Robinson, G. et al. Novel mutations target distinct subgroups of medulloblastoma. Nature 488, 43-48 (2012).

50. Parsons, D. W. et al. The genetic landscape of the childhood cancer medulloblastoma. Science 331 435-439 (2011).

This is the first exome-level sequencing study of $M B$, identifying novel recurrent mutations in the chromatin-modifying genes MLL2 and MLL3.

51. Canning, P. et al. Structural basis for Cul3 protein assembly with the BTB-Kelch family of E3 ubiquitin ligases. J. Biol. Chem. 288, 7803-7814 (2013).

52. Lee, J. C. et al. Recurrent KBTBD4 small in-frame insertions and absence of DROSHA deletion or DICER 1 mutation differentiate pineal parenchymal tumor of intermediate differentiation (PPTID) from pineoblastoma. Acta Neuropathol. 137, 851-854 (2019).

53. Northcott, P. A. et al. Enhancer hijacking activates GFI1 family oncogenes in medulloblastoma. Nature 511, 428-434 (2014).

This study identifies recurrent structural variations leading to the activation of GFI1 and GFI1 B in Group $3 \mathrm{MB}$, without affecting the protein sequence of these oncoproteins.

54. Moroy, T., Vassen, L., Wilkes, B. \& Khandanpour, C. From cytopenia to leukemia: the role of Gfi 1 and $\mathrm{Gfi} 1 \mathrm{~b}$ in blood formation. Blood 126, 2561-2569 (2015).

55. Erikson, J. et al. Translocation of an immunoglobulin kappa locus to a region 3' of an unrearranged c-myc oncogene enhances c-myc transcription. Proc. Natl Acad. Sci. USA 80, 7581-7585 (1983).

56. Croce, C. M. et al. Transcriptional activation of an unrearranged and untranslocated c-myc oncogene by translocation of a $\mathrm{C}$ lambda locus in Burkitt. Proc. Natl Acad. Sci. USA 80, 6922-6926 (1983)

57. Haller, F. et al. Enhancer hijacking activates oncogenic transcription factor NR4A3 in acinic cell carcinomas of the salivary glands. Nat. Commun. 10, 368 (2019).

58. Martin-Garcia, D. et al. CCND2 and CCND3 hijack immunoglobulin light-chain enhancers in cyclin D1 (-) mantle cell lymphoma. Blood 133, 940-951 (2019).

59. Zimmerman, M. W. et al. MYC drives a subset of high-risk pediatric neuroblastomas and is activated through mechanisms including enhancer hijacking and focal enhancer amplification. Cancer Discov. 8 320-335 (2018).

60. Weischenfeldt, J. et al. Pan-cancer analysis of somatic copy-number alterations implicates IRS 4 and IGF2 in enhancer hijacking. Nat. Genet. 49, 65-74 (2017).

61. Ryan, R. J. et al. Detection of enhancer-associated rearrangements reveals mechanisms of oncogene dysregulation in B-cell lymphoma. Cancer Discov. 5 , 1058-1071 (2015).

62. Wu, Y. et al. PRDM6 is enriched in vascular precursors during development and inhibits endothelial cell proliferation, survival, and differentiation. J. Mol. Cell. Cardiol. 44, 47-58 (2008).

63. Davis, C. A. et al. PRISM/PRDM6, a transcriptional repressor that promotes the proliferative gene program in smooth muscle cells. Mol. Cell. Biol. 26, 2626-2636 (2006).

64. Hunger, S. P. \& Mullighan, C. G. Acute lymphoblastic leukemia in children. N. Engl. J. Med. 373, 1541-1552 (2015).

65. Parker, M. et al. C11 orf95-RELA fusions drive oncogenic NF-kB signalling in ependymoma. Nature 506, 451-455 (2014).

66. Jones, D. T. et al. Tandem duplication producing a novel oncogenic BRAF fusion gene defines the majority of pilocytic astrocytomas. Cancer Res. 68 8673-8677 (2008).

67. He, F. et al. Long noncoding RNA PVT1-214 promotes proliferation and invasion of colorectal cancer by stabilizing Lin28 and interacting with miR-128. Oncogene 38, 164-179 (2019).

68. Liu, Z. \& Zhang, H. LncRNA plasmacytoma variant translocation 1 is an oncogene in bladder urothelial carcinoma. Oncotarget 8, 64273-64282 (2017).

69. Tian, Z. et al. LncRNA PVT 1 regulates growth, migration, and invasion of bladder cancer by miR-31/ CDK1. J. Cell. Physiol. 234, 4799-4811 (2019).

70. Tseng, Y. Y. et al. PVT1 dependence in cancer with MYC copy-number increase. Nature 512, 82-86 (2014).

71. Wang, Z., Su, M., Xiang, B., Zhao, K. \& Oin, B. Circular RNA PVT1 promotes metastasis via miR-145 sponging in CRC. Biochem. Biophys. Res. Commun 512, 716-722 (2019).

72. Yang, T. et al. IncRNA PVT1 and its splicing variant function as competing endogenous RNA to regulate 
clear cell renal cell carcinoma progression. Oncotarget 8, 85353-85367 (2017)

73. Zhao, J. et al. LncRNA PVT1 promotes angiogenesis via activating the STAT3/VEGFA axis in gastric cancer. Oncogene 37, 4094-4109 (2018).

74. Cho, S. W. et al. Promoter of IncRNA gene PVT1 is a tumor-suppressor DNA boundary element. Cell 173 1398-1412.e22 (2018).

75. Hovestadt, V. et al. Decoding the regulatory landscape of medulloblastoma using DNA methylation sequencing. Nature 510, 537-541 (2014).

76. Balzeau, J., Menezes, M. R., Cao, S. \& Hagan, J. P. The LIN28/let-7 pathway in cancer. Front. Genet. 8 , 31 (2017)

77. Northcott, P. A. et al. The miR-17/92 polycistron is upregulated in sonic hedgehog-driven medulloblastomas and induced by $\mathrm{N}$-myc in sonic hedgehog-treated cerebellar neural precursors. Cancer Res. 69 3249-3255 (2009)

78. Ferretti, E. et al. MicroRNA profiling in human medulloblastoma. Int. J. Cancer 124, 568-577 (2009).

79. Uziel, T. et al. The miR-17 92 cluster collaborates with the Sonic Hedgehog pathway in medulloblastoma. Proc. Natl Acad. Sci. USA 106, 2812-2817 (2009).

80. Zindy, F. et al. Role of the miR-17 approximately 92 cluster family in cerebellar and medulloblastoma development. Biol. Open 3, 597-605 (2014).

81. Murphy, B. L. et al. Silencing of the miR-17 92 cluster family inhibits medulloblastoma progression. Cancer Res. 73, 7068-7078 (2013).

82. Bai, A. H. et al. MicroRNA-182 promotes leptomeningeal spread of non-sonic hedgehogmedulloblastoma. Acta Neuropathol. 123, 529-538 (2012).

83. Weeraratne, S. D. et al. Pleiotropic effects of miR$183 \sim 96 \sim 182$ converge to regulate cell survival, proliferation and migration in medulloblastoma. Acta Neuropathol. 123, 539-552 (2012).

84. Zhang, Z., Li, S. \& Cheng, S. Y. The miR-183 approximately 96 approximately 182 cluster promotes tumorigenesis in a mouse model of medulloblastoma. J. Biomed. Res. 27, 486-494 (2013)

85. Ferretti, E. et al. Concerted microRNA control of Hedgehog signalling in cerebellar neuronal progenitor and tumour cells. EMBO J. 27, 2616-2627 (2008).

86. Su, X. et al. Abnormal expression of REST/NRSF and Myc in neural stem/progenitor cells causes cerebellar tumors by blocking neuronal differentiation Mol. Cell. Biol. 26, 1666-1678 (2006).

87. Wu, J. \& Xie, X. Comparative sequence analysis reveals an intricate network among REST, CREB and miRNA in mediating neuronal gene expression. Genome Biol. 7 , R85 (2006).

88. Pierson, J., Hostager, B., Fan, R. \& Vibhakar, R. Regulation of cyclin dependent kinase 6 by microRNA 124 in medulloblastoma. J. Neurooncol. 90, 1-7 (2008).

89. Silber, J. et al. Expression of miR-124 inhibits growth of medulloblastoma cells. Neuro-Oncology 15, 83-90 (2013).

90. Wang, X. et al. miR miR on the wall, who's the most malignant medulloblastoma miR of them all? Neuro Oncology 20, 313-323 (2018)

91. Hansen, K. D. et al. Increased methylation variation in epigenetic domains across cancer types. Nat. Genet. 43, 768-775 (2011).

92. Berman B. P. et al. Regions of focal DNA hypermethylation and long-range hypomethylation in colorectal cancer coincide with nuclear laminaassociated domains. Nat. Genet. 44, 40-46 (2011).

93. Hon, G. C. et al. Global DNA hypomethylation coupled to repressive chromatin domain formation and gene silencing in breast cancer. Genome Res. 22, 246-258 (2012)

94. Lin, C. Y. et al. Active medulloblastoma enhancers reveal subgroup-specific cellular origins. Nature $\mathbf{5 3 0}$ 57-62 (2016)

95. Consortium, E. P. An integrated encyclopedia of DNA elements in the human genome. Nature 489, 57-74 (2012).

96. Thurman, R. E. et al. The accessible chromatin landscape of the human genome. Nature 489, 75-82 (2012).

97. Roadmap Epigenomics Consortium et al. Integrative analysis of 111 reference human epigenomes. Nature 518, 317-330 (2015).

98. Aebersold, R \& Mann, M Mass-spectrometric exploration of proteome structure and function. Nature 537, 347-355 (2016)

99. Archer, T. C. et al. Proteomics, post-translational modifications, and integrative analyses reveal molecular heterogeneity within medulloblastoma subgroups. Cancer Cell 34, 396-410 e398 (2018)

100. Forget, A. et al. Aberrant ERBB4-SRC signaling as a hallmark of group 4 medulloblastoma revealed by integrative phosphoproteomic profiling. Cancer Cell 34, 379-395.e377 (2018)

Archer et al. (2018) and Forget et al. (2018) first describe quantification of the global proteome and phospho-proteome landscape in primary patient samples through mass spectrometry.

101. Rivero-Hinojosa, S. et al. Proteomic analysis of medulloblastoma reveals functional biology with translational potential. Acta Neuropathol. Commun. 6 48 (2018)

102. Vogel, C. \& Marcotte, E. M. Insights into the regulation of protein abundance from proteomic and transcriptomic analyses. Nat. Rev. Genet. 13, 227-232 (2012)

103. Staal, J. A. et al. Proteomic profiling of high risk medulloblastoma reveals functional biology. Oncotarget 6, 14584-14595 (2015)

104. Zomerman, W. W. et al. Identification of two proteinsignaling states delineating transcriptionally heterogeneous human medulloblastoma. Cell Rep. 22 3206-3216 (2018)

105. Canettieri, G. et al. Histone deacetylase and Cullin3-REN(KCTD11) ubiquitin ligase interplay regulates Hedgehog signalling through Cli acetylation. Nat. Cell Biol. 12, 132-142 (2010)

106. Klisch, T. J., Vainshtein, A., Patel, A. J. \& Zoghbi, H. Y. Jak2-mediated phosphorylation of Atoh1 is critical for medulloblastoma growth. eLife 6, 31181 (2017).

107. Al-Hajj, M., Wicha, M. S., Benito-Hernandez, A., Morrison, S. J. \& Clarke, M. F. Prospective identification of tumorigenic breast cancer cells. Proc. Natl Acad. Sci. USA 100, 3983-3988 (2003)

108. Lapidot, T. et al. A cell initiating human acute myeloid leukaemia after transplantation into SCID mice. Nature 367, 645-648 (1994).

109. Bonnet, D. ¿ Dick, J. E. Human acute myeloid leukemia is organized as a hierarchy that originates from a primitive hematopoietic cell. Nat. Med. 3 , 730-737 (1997).

110. Singh, S. K. et al. Identification of a cancer stem cell in human brain tumors. Cancer Res. 63, 5821-5828 (2003).

111. Singh, S. K. et al. Identification of human brain tumour initiating cells. Nature 432, 396-401 (2004). This study first reports the discovery of a CD133 stem-like tumour cell population in MB that can efficiently initiate tumours in vivo, whereas the remaining CD133-tumour cells cannot.

112. Garg, N. et al. CD133+ brain tumor-initiating cells are dependent on STAT3 signaling to drive medulloblastoma recurrence. Oncogene 36, 606-617 (2017).

113. Read, T. A. et al. Identification of CD15 as a marker for tumor-propagating cells in a mouse model of medulloblastoma. Cancer Cell 15, 135-147 (2009).

114. Ward, R. J. et al. Multipotent CD15 + cancer stem cells in patched-1-deficient mouse medulloblastoma. Cancer Res. 69, 4682-4690 (2009).

115. Vanner, R. J. et al. Quiescent sox $2^{+}$cells drive hierarchical growth and relapse in sonic hedgehog subgroup medulloblastoma. Cancer Cell 26, 33-47 (2014).

116. Weng, Q. et al. Single-cell transcriptomics uncovers glial progenitor diversity and cell fate determinants during development and gliomagenesis. Cell Stem Cell 24, 707-723.e708 (2019).

117. Filbin, M. G. et al. Developmental and oncogenic programs in H3K27M gliomas dissected by single-cell RNA-seq. Science 360, 331-335 (2018).

118. Venteicher, A. S. et al. Decoupling genetics, lineages, and microenvironment in IDH-mutant gliomas by single-cell RNA-seq. Science 355, eaai8478 (2017).

119. Tirosh, I. et al. Single-cell RNA-seq supports a developmental hierarchy in human oligodendroglioma. Nature 539, 309-313 (2016)

120. Patel, A. P. et al. Single-cell RNA-seq highlights intratumoral heterogeneity in primary glioblastoma. Science 344, 1396-1401 (2014)

121. Vladoiu, M. C. et al. Childhood cerebellar tumours mirror conserved fetal transcriptional programs. Nature 572, 67-73 (2019).

122. Hovestadt, V. et al. Resolving medulloblastoma cellular architecture by single-cell genomics. Nature 572, 74-79 (2019)

Vladoiu et al. (2019) and Hovestadt et al. (2019) first apply single-cell transcriptome profiling to characterizing cellular heterogeneity within individual patients with MB and identifying developmental correlates from the developing mouse cerebellum.
123. Oliver, T. G. et al. Loss of patched and disruption of granule cell development in a pre-neoplastic stage of medulloblastoma. Development 132, 2425-2439 (2005).

124. Morrissy, A. S. et al. Spatial heterogeneity in medulloblastoma. Nat. Genet. 49, 780-788 (2017).

125. Fults, D. W., Taylor, M. D. \& Garzia, L. Leptomeningea dissemination: a sinister pattern of medulloblastoma growth. J. Neurosurg. https://doi.org/10.3171/ 2018.11.PEDS18506 (2019).

126. Ramaswamy, V. et al. Recurrence patterns across medulloblastoma subgroups: an integrated clinical and molecular analysis. Lancet Oncol. 14, 1200-1207 (2013).

127. Hill, R. M. et al. Combined MYC and P53 defects emerge at medulloblastoma relapse and define rapidly progressive, therapeutically targetable disease. Cancer Cell 27, 72-84 (2015)

128. Morrissy, A. S. et al. Divergent clonal selection dominates medulloblastoma at recurrence. Nature 529, 351-357 (2016).

129. Zapotocky, M. et al. Differential patterns of metastatic dissemination across medulloblastoma subgroups. J. Neurosurg. 21, 145-152 (2018).

130. Garzia, L. et al. A hematogenous route for medulloblastoma leptomeningeal metastases. Cell 173, 1549 (2018) This study describes the presence of circulating tumour cells in the blood of patients that can spread to form leptomeningeal metastases.

131. US National Library of Medicine. ClinicalTrials.gov https://clinicaltrials.gov/ct2/show/NCT01878617 (2013).

132. US National Library of Medicine. ClinicalTrials.gov https://clinicaltrials.gov/ct2/show/NCT02066220 (2014).

133. US National Library of Medicine. ClinicalTrials.gov https://clinicaltrials.gov/ct2/show/NCT02724579 (2016).

134. Sharma, T. et al. Second-generation molecular subgrouping of medulloblastoma: an international meta-analysis of Group 3 and Group 4 subtypes. Acta Neuropathol. 138, 309-326 (2019).

135. Druker, H. et al. Genetic counselor recommendations for cancer predisposition evaluation and surveillance in the pediatric oncology patient. Clin. Cancer Res. $\mathbf{2 3}$ e91-e97 (2017).

136. Romer, J. T. et al. Suppression of the Shh pathway using a small molecule inhibitor eliminates medulloblastoma in Ptc $1^{+/-} p 53^{-/-}$mice. Cancer Cell 6 . 229-240 (2004).

137. Lee, Y. et al. Loss of suppressor-of-fused function promotes tumorigenesis. Oncogene 26, 6442-6447 (2007).

138. Robinson, G. W. et al. Vismodegib exerts targeted efficacy against recurrent sonic hedgehog-subgroup medulloblastoma: results from phase II Pediatric Brain Tumor Consortium studies PBTC-025B and PBTC-032. J. Clin. Oncol. 33, 2646-2654 (2015).

139. Robinson, G. W. et al. Irreversible growth plate fusions in children with medulloblastoma treated with a targeted hedgehog pathway inhibitor. Oncotarget 8 69295-69302 (2017)

140. Kimura, H., Ng, J. M. \& Curran, T. Transient inhibition of the Hedgehog pathway in young mice causes permanent defects in bone structure. Cancer Cell 13 249-260 (2008)

141. Lee, C. et al. Lsd 1 as a therapeutic target in Gfi1activated medulloblastoma. Nat. Commun. 10, 332 (2019).

142. Morabito, $M$ et al. An autocrine ActivinB mechanism drives TGF $\beta$ /activin signaling in Group 3 medulloblastoma. EMBO Mol. Med. 11, e9830 (2019).

143. Gilbertson, R. J. \& Ellison, D. W. The origins of medulloblastoma subtypes. Annu. Rev. Pathol. 3 341-365 (2008)

144. Gibson, P. et al. Subtypes of medulloblastoma have distinct developmental origins. Nature $\mathbf{4 6 8}$, 1095-1099 (2010).

145. Swartling, F. J. et al. Distinct neural stem cell populations give rise to disparate brain tumors in response to N-MYC. Cancer Cell 21, 601-613 (2012).

146. Pei, Y. et al. An animal model of MYC-driven medulloblastoma. Cancer Cell 21, 155-167 (2012).

147. Kawauchi, D. et al. Novel MYC-driven medulloblastoma models from multiple embryonic cerebellar cells. Oncogene 36, 5231-5242 (2017).

148. Kawauchi, D. et al. A mouse model of the most aggressive subgroup of human medulloblastoma. Cancer Cell 21, 168-180 (2012). 
149. Swartling, F. J. et al. Pleiotropic role for $\mathrm{MYCN}$ in medulloblastoma. Genes Dev. 24, 1059-1072 (2010).

150. Chizhikov, V. V. et al. Lmx1 a regulates fates and location of cells originating from the cerebellar rhombic lip and telencephalic cortical hem. Proc. Natl Acad. Sci. USA 107, 10725-10730 (2010).

151. Englund, C. et al. Unipolar brush cells of the cerebellum are produced in the rhombic lip and migrate through developing white matter. J. Neurosci. 26, 9184-9195 (2006)

\section{Acknowledgements}

P.A.N. is a Pew-Stewart Scholar for Cancer Research (Margaret and Alexander Stewart Trust) and recipient of the Sontag Foundation Distinguished Scientist Award. P.A.N was also supported by the National Cancer Institute
(R01CA232143-01), the American Association for Cancer Research (NextGen Grant for Transformative Cancer Research) The Brain Tumour Charity (Quest for Cures and Clinical Biomarkers), the American Lebanese Syrian Associated Charities (ALSAC) and St. Jude. V.H. is supported by a Human Frontier Science Program long-term fellowship (LT000596/2016-L). We acknowledge K. Smith, L. Bihannic and T. Sharma for assistance with data organization and figure design. We thank B. Stelter for assistance with the artwork.

\section{Author contributions}

V.H. and P.A.N researched data for the article, contributed substantially to discussion of the content, wrote the article and reviewed/edited the manuscript before submission. O.A. F.J.S., G.W.R. and S.M.P. researched data for the article, wrote the article and reviewed/edited the manuscript before submission.

\section{Competing interests}

The authors declare no competing interests.

\section{Peer review information}

Nature Reviews Cancer thanks T. Curran, E. Ferretti and the other, anonymous, reviewer(s) for their contribution to the peer review of this work.

\section{Publisher's note}

Springer Nature remains neutral with regard to jurisdictional claims in published maps and institutional affiliations.

(c) Springer Nature Limited 2019 